University of Redlands

\title{
Examining Hotspots of Traffic Collisions in Redlands, California
}

A Major Individual Project submitted in partial satisfaction of the requirements for the degree of Master of Science in Geographic Information Systems

by

Steven David Bills

Mark Kumler, Ph.D., Committee Chair

Fang Ren, Ph.D.

December 2009 
Examining Hotspots of Traffic Collisions in Redlands, California

Copyright (c) 2009

by

Steven David Bills 
The report of Steven David Bills is approved.

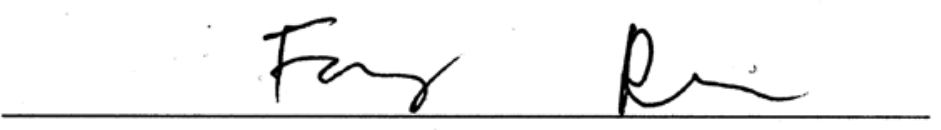

Fang Ren, Ph.D.

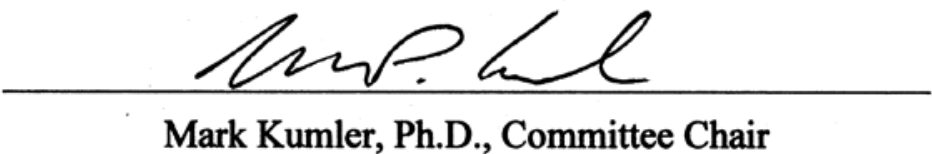

December 2009 



\section{Acknowledgements}

There are many people who have helped me in the MS GIS program who I would like to thank. I would like to thank Mark Kumler, my advisor, for helping me figure out what my project was really about and for pushing me to get it done. I would also like to thank Fang Ren and Douglas Flewelling, who both gave me advice and help with this project. There is no way that I could have gotten through this program without the help of the rest of the faculty and staff in the MS GIS program, especially Debbie Riley.

I would like to thank my fellow students in the program. They have provided a great support system for me throughout the past year and I could not have done it without them. I would also like to thank my family and friends for their support over the past year. 



\author{
Abstract \\ Examining Hotspots of Traffic Collisions in Redlands, California \\ by \\ Steven David Bills
}

Traffic collisions are a major concern for the government agencies responsible for our road networks. Knowing where areas with high densities of traffic collision are gives the agencies guidance in where to best implement traffic collision reduction measures. This project explains how ModelBuilder, a modeling tool, was used to determine hotspots of traffic collisions in the City of Redlands. Using kernel density estimation, raster reclassification, and raster to polygon conversion, the model creates hotspot polygons using traffic collision point data as input. Traffic collision attribute data is then added to the hotspot polygons. A web application was also developed for examining the hotspots and their attribute data. This web application provides the city with a user friendly interface for examining the hotspots and can help the city determine which traffic collision reduction measures should be implemented where. 



\section{Table of Contents}

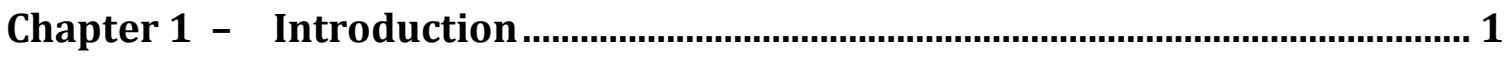

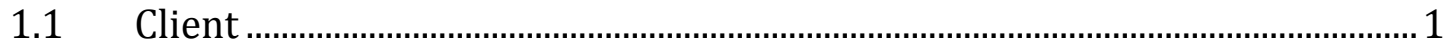

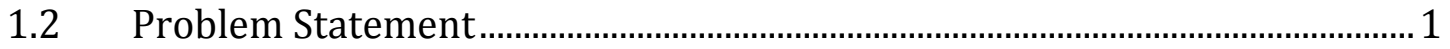

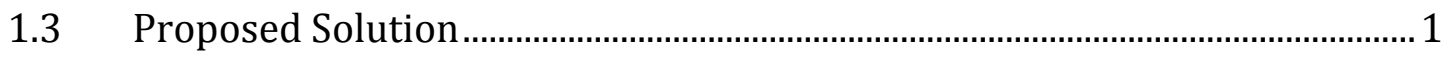

1.3.1 Goals and Objectives …………………………………………………….... 2

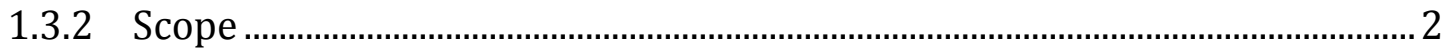

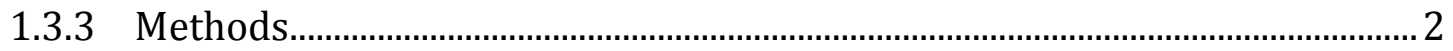

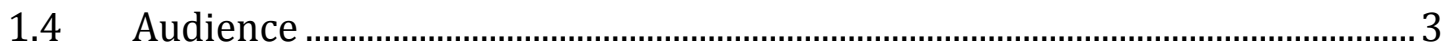

1.5 Overview of the Rest of this Report ................................................................... 3

Chapter 2 - Background and Literature Review................................................ 5

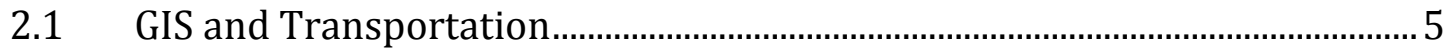

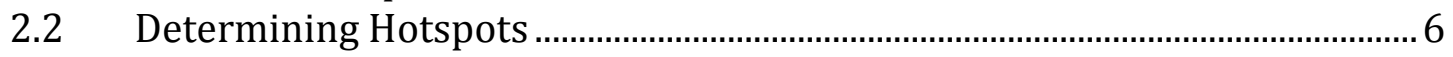

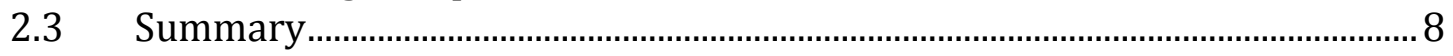

Chapter 3 - Systems Analysis and Design …………........................................11

$3.1 \quad$ Problem Statement......................................................................................... 11

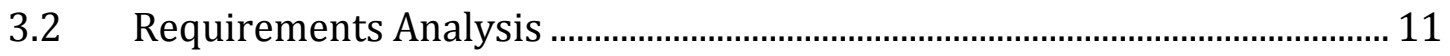

3.2.1 Functional Requirements............................................................................... 11

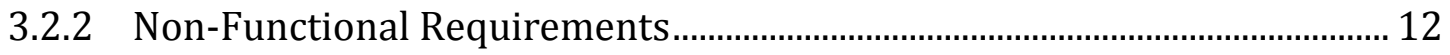

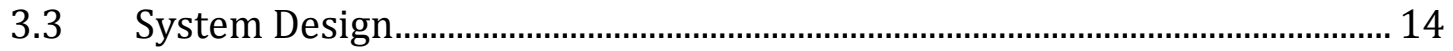

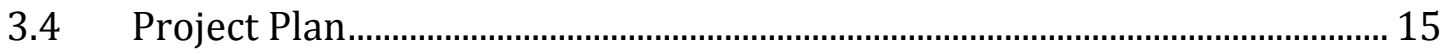

3.4.1 Requirements Analysis .................................................................................. 16

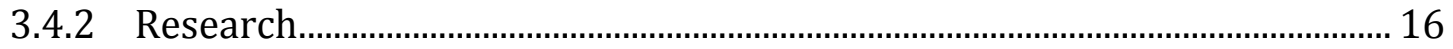

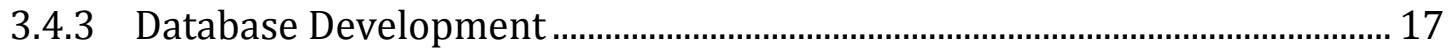

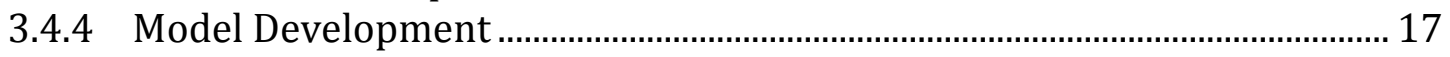

3.4.5 Web Application Development ........................................................................ 17

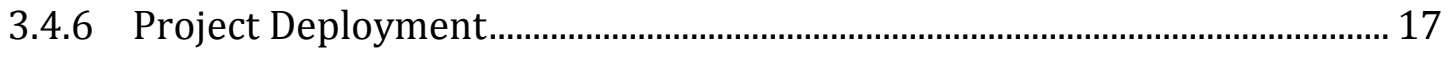

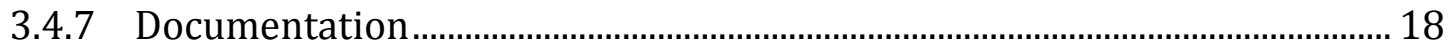

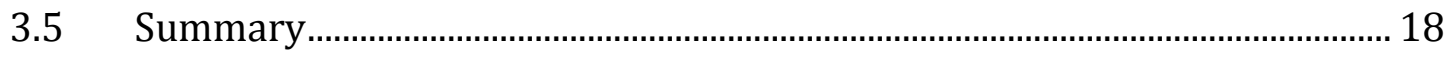

Chapter 4 - Database Design .........................................................................19

4.1 Conceptual Data Model .............................................................................. 19

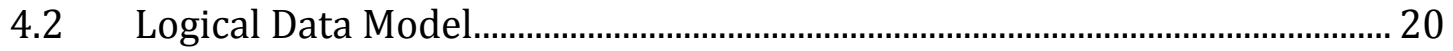

4.2.1 Collision Hotspot File Geodatabase.................................................................. 20

4.2.2 Collision Feature Class ................................................................................ 21

4.2.3 Traffic Signal and Stop Sign Feature Classes ................................................ 21

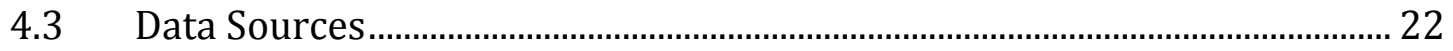

4.4 Data Scrubbing and Loading .......................................................................... 22

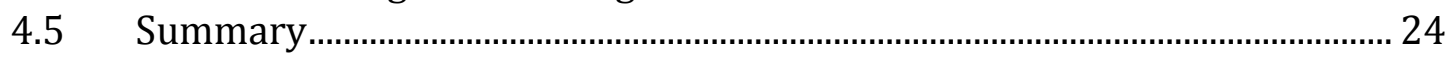

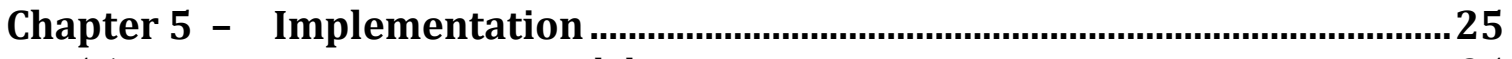

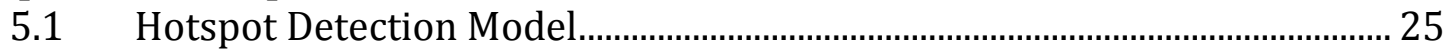

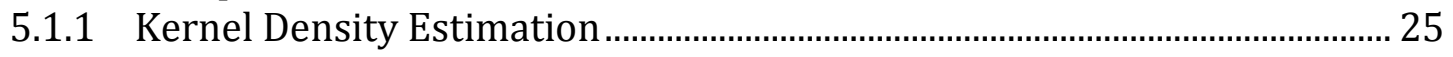

5.1.2 Reclassification................................................................................................. 29 
5.1.3 Adding Collision Attribute Data ........................................................................ 32

$5.2 \quad$ Hotspot Exploration Web Application ……………………………………..... 32

5.2.1 Data Layers ……………………………………………………………….... 32

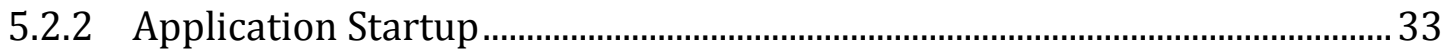

5.2.3 Hotspot Exploration Tools ................................................................................. 33

5.2.4 Hotspot Exploration Tool Output....................................................................... 34

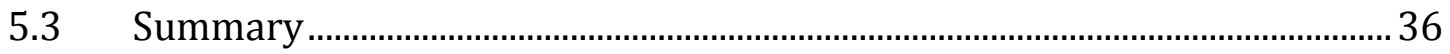

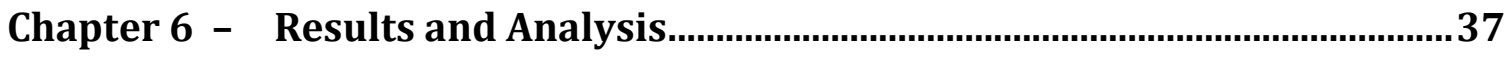

6.1 Hotspot Detection Model Results...................................................................37

6.2 Hotspot Exploration Web Application …………………………………….... 41

6.3 Implementation on the Client's Network ........................................................ 45

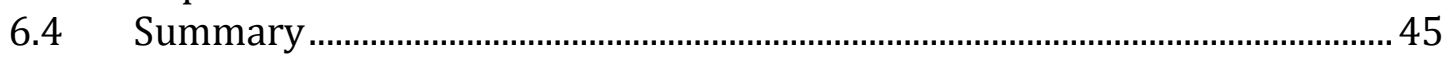

Chapter 7 - Conclusion and Future Work ……….............................................47

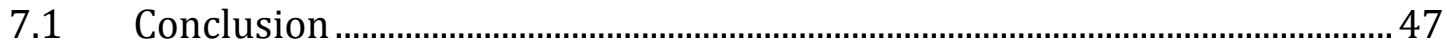

7.2 Future Work ......................................................................................................... 48

7.2.1 Accurate Traffic Collision Point Data .................................................................. 48

7.2.2 Traffic Volume Data .......................................................................................... 48

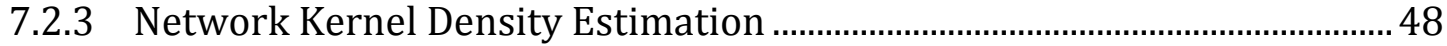

7.2.4 Simulation to Determine Statistical Significance ........................................... 48

7.2.5 Additions to the Web Application ...................................................................... 49

Works Cited 51

Appendix A. Web Application Code....................................................................53 


\section{Table of Figures}

Figure 1.1 Location of San Bernardino County within California....................... 1

Figure 1.2 Location of Redlands within San Bernardino County ........................ 1

Figure 3.1 Project Plan Tasks............................................................................ 16

Figure 4.1 Conceptual Data Model .................................................................20

Figure 4.2 Collision Feature Class .............................................................. 21

Figure 4.3 Traffic Signal and Stop Sign Feature Classes.................................22

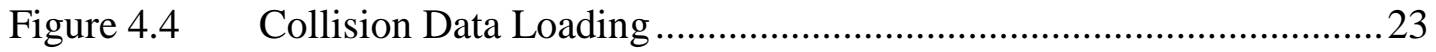

Figure 4.5 Calculation for Converting Fields to Proper Case .............................24

Figure 5.1 Complete Hotspot Detection Model ..................................................25

Figure 5.2 Kernel Density Estimation Step in the Model ..................................26

Figure 5.3 Bandwidth Selections for Kernel Density.......................................27

Figure 5.4 Cell Size Selections for Kernel Density........................................28

Figure 5.5 Reclassification Step in the Model ..................................................29

Figure 5.6 Reclassification Methods for Kernel Density ................................... 31

Figure 5.7 Adding Collision Attribute Data Step in the Model ........................... 32

Figure 5.8 Web Application Tool Procedures.................................................... 34

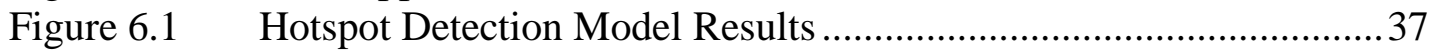

Figure 6.2 Hotspot Polygon Attribute Table ...................................................39

Figure 6.3 Web Application Base Maps ............................................................. 42

Figure 6.4 Traffic Signal and Stop Sign Display ............................................. 43

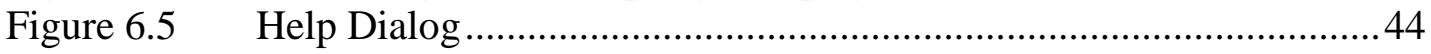

Figure 6.6 Table and Chart in Tool Output .................................................... 44 



\section{List of Tables}

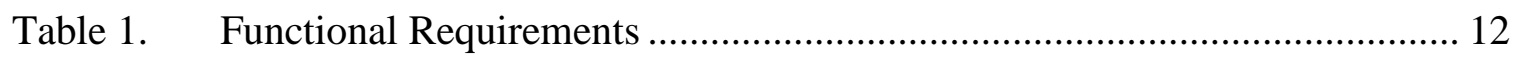

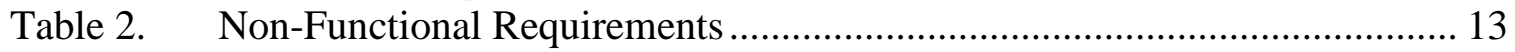

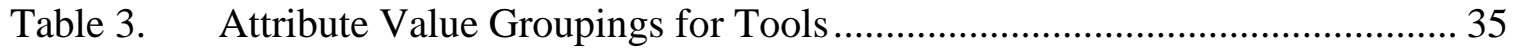

Table 4. Hotspot Polygons Not Covering Road Network.......................................... 40 



\section{List of Acronyms and Definitions}

$\begin{array}{ll}\text { API } & \text { Application Programming Interface } \\ \text { ESRI } & \text { Environmental Systems Research Institute } \\ \text { GIS } & \text { Geographic Information Systems } \\ \text { GPS } & \text { Global Positioning Systems } \\ \text { GUI } & \text { Graphical User Interface } \\ \text { KDE } & \text { Kernel Density Estimation }\end{array}$





\section{Chapter 1 - Introduction}

Vast road networks exist across the modern world. These road networks have many advantages. They allow for the quick movement of both people and goods across cities and countries. They have greatly increased the connectedness of the world. Unfortunately these road networks also have negative effects. Traffic collisions are an all too common occurrence on the roads. Traffic collisions are a major concern since they can cause injury or the loss of life. The World Health Organization states that traffic collisions are the leading injury related cause of death among people age $10-24$ (Anderson, 2009). The reduction of traffic collisions is a major concern of the agencies that govern the road networks. In order for agencies to implement measures to reduce the number of traffic collisions they first need to know where traffic collisions are frequently occurring.

This project proposed a method for determining the locations of areas with high densities of traffic collisions, also called hotspots. This project utilized geographic information systems (GIS) for analyzing the locations of traffic collisions to determine hotspots. An application for viewing the hotspots was also developed as a part of this project. The tools provided as part of this application allow the user to view the attributes of the collisions that occurred in each hotspot. This information will help to determine where traffic collision reduction measures should be implemented and what type of measures should be used.

This chapter first introduces the client for this project and the problem that was addressed. The proposed solution for that problem is discussed, including the goals and objectives of the project, the scope of the project, and the methodology used. The intended audience of this report is then detailed. Finally, the contents of the rest of the report are summarized.

\subsection{Client}

The client for this project was the City of Redlands. The City of Redlands is located in the southwest portion of San Bernardino County in California (Figures 1.1 \& 1.2). As of January, 2007, the City of Redlands had a population of 71,375 (City of Redlands Redevelopment Agency, 2009). The City of Redlands Traffic and Parking Commission is in charge of traffic studies in the city and keeps records of traffic collisions within the city. They currently use a pin map for plotting traffic collisions. 



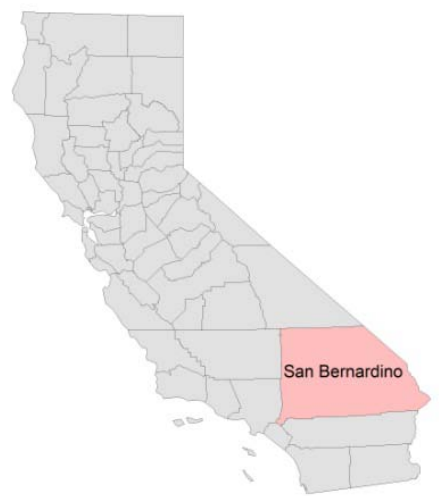

Figure 1.1 Location of San Bernardino County within California

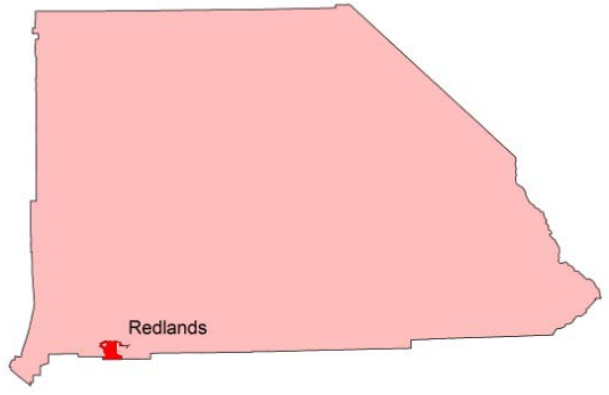

Figure 1.2 Location of Redlands within San Bernardino County

The point of contact for the project was Phil Mielke, the GIS Administrator for the City of Redlands. He supported this project by:

- Providing city infrastructure data

- Aiding in acquiring traffic accident data for the city

- Providing input on analysis type and output

\subsection{Problem Statement}

Traffic accidents are a common problem in any transportation network, and are compounded by the fact that traffic accidents often result in injury or the loss of life. The reduction of traffic collisions is a goal of transportation divisions at all levels of government. The City of Redlands would like to determine where high numbers of traffic accidents are occurring within the city. Determining areas with high numbers of traffic accidents will allow the city to concentrate on the areas of highest risk for traffic accident reduction. The city would also like to find any patterns or common attributes among the areas of high traffic accidents. Knowing any common attributes or patterns in these areas can help them understand why certain types of traffic accidents are occurring at certain locations. This will help the city in determining the most effective traffic collision reducing measures for each area.

\subsection{Proposed Solution}

This project developed a method for determining traffic collision hotspot locations using GIS. The results of the analysis were used in a web application that allows a user to examine the attributes associated with individual hotspots. 


\subsubsection{Goals and Objectives}

The first goal of the project was to determine traffic collision hotspot locations within the City of Redlands by using GIS and adding important attribute data to the hotspots. The analytical abilities of GIS proved useful in accomplishing this goal. The second goal was to provide an application for viewing and examining the hotspots and their attributes. Tools within the application would provide the functionality for viewing the information on individual hotspots.

The objectives for this project included:

- Designing a database for the traffic collision data

- Creating a model to determine hotspots of traffic accidents

- Assigning attributes to the hotspots

- Creating a web application to view the hotspots

- Providing tools in the application for examining hotspot attribute data

\subsubsection{Scope}

Project limitations were set according to the requirements of the client and the time limitations of the project. They included the spatial extent, amount of data used, complexity of the analysis model, and the complexity of the web application.

The spatial extent of the project was limited to the City of Redlands boundaries, given that this is the area of importance to the client. This limitation was also beneficial in reducing the complexity of the project.

The traffic collision data was limited to a two and a half year period. The temporal coverage of the data is from January 1, 2007 through July 18, 2009. The data was also limited to traffic collisions that occurred on non-interstate roads in the City of Redlands. Interstate roads are maintained at the state level and are not of interest to the client.

The complexity of the model used for analysis in the project was limited due to the time frame of the project. The input data for the model was limited to the traffic collision point data received from the client. The number of methods and processes used in the model were also limited to reduce complexity to allow the project to be completed in the required timeframe.

The complexity of the web application was also limited due to the time frame, to increase the user friendliness of the application. The number of tools for examining hotspot attributes provided in the application was limited to eight: month of the year, time of day, severity, collision type, light condition, weather condition, number of injuries, and number of fatalities. The output of the tools was limited to a graphical output of a table and column chart of the results.

\subsubsection{Methods}

The methodology of the project was broken into multiple steps. The steps are as follows:

1. Create a collision density surface using kernel density estimation.

2. Reclassify the surface to determine hotspots.

3. Convert the hotspots to polygons and add collision attribute data.

4. Develop a web application for examining the hotspots and their attributes. 


\subsection{Audience}

The intended audience for this paper is professionals with a background in GIS and a basic understanding of statistics and spatial analysis. The end users of this project will be employees of the City of Redlands, specifically those on the Traffic and Parking Commission. While the end users will benefit from this project, it is not expected that they will have knowledge of GIS and therefore are not included in the intended audience.

\subsection{Overview of the Rest of this Report}

The rest of the report will follow the workflow that was used for the project. Chapter 2 will discuss the project background, as well as the literature reviewed for the project. Chapter 3 will go over the system design, including the requirements analysis and the plan for the project. Chapter 4 will go over the database design, including the data collection and data loading and scrubbing. Chapter 5 will discuss the implementation of the project, including the development of the models and the web application. Chapter 6 will focus on the results and analysis of the models and web application. Chapter 7 will go over the conclusion gained from the project and future work to be done in the project area. 



\section{Chapter 2 - Background and Literature Review}

Traffic collisions are a major public safety issue. Before measures can be taken to reduce the number and severity of traffic collision, engineers need to know where to look to most effectively make proper changes. This chapter discusses the increasing use of GIS and GIS-related technology in the transportation industry. The importance of examining traffic collisions and traffic collision hotspots is also discussed. After giving a definition of a hotspot, methods for determining hotspots are considered, so an appropriate method for this project can be implemented.

\subsection{GIS and Transportation}

As GIS technology has increased over time, it has become increasingly implemented in the transportation industry. Lang (1999) discusses various ways that GIS is being implemented in the transportation industry. Some of these areas include highway design, highway repair, fleet management, public transportation planning, and traffic control. Lang also points out that with this increase there have also been a wider variety of instruments and tools used for data collection and processing. In-pavement sensors, closed-caption television, and red-light cameras are used to collect traffic and speed data that can be analyzed by GIS. Global positioning systems (GPS) are used in vehicles for data collection and vehicle navigation. GIS is a fundamental tool in researching transportation because it is capable of efficient analysis and visualization of spatial data (Anderson, 2009). Lang (1999) notes that the increase in internet access has been a benefit to the use of GIS in the transportation industry. "Public access via the Internet will allow spatially enabled transportation databases to be made available to a majority of citizens" (Lang, 1999, p. 9).

One of the most common uses of GIS in the transportation industry is the analysis of traffic collisions (Lang, 1999). Lang notes that GIS can answer questions like which intersections have the most collisions, which kind of collisions occur most frequently at a location, and what time of day collisions usually occur at a location. Being able to answer these questions can lead to the implementation of measures to reduce the number of collisions. Anderson (2009) states that the impact of traffic collisions is a social and public health challenge due to resulting injuries and fatalities. Anderson also notes that, according to the World Health Organization, traffic collisions are the leading injury related cause of death among people aged $10-24$. This highlights the importance of analyzing traffic collisions using GIS to help reduce collision rates. According to Steenberghen, Dufays, Thomas, and Flahaut (2004), the examination of spatial patterns of traffic collisions is the most straightforward use of GIS for accident analysis. "An improved understanding of spatial patterns of traffic accidents can make accident reduction efforts more effective. For instance, by knowing where and when traffic accidents usually occur, law enforcement can conduct more efficient patrols and highway departments can disseminate more effectively to drivers the critical information about roadway conditions" (Xie \& Yan 2008, p.4). Lang (1999) also points out that traffic collisions happen for many different reasons. When they start to occur frequently and at the same location, causes other than careless drivers are suspected. Lang states that these other causes could include narrow roads, tight corners, intersections with no turn lanes, 
and traffic lights that go from red to green before the intersection clears. The analysis of traffic collisions with GIS gives traffic engineers a starting point for any necessary changes to the roadway (Lang, 1999).

When performing analysis of traffic collisions, it is important to look at patterns and groupings of collisions and not only look at individual collisions. Anderson (2009) states that considering traffic collisions only as points on a map isn't robust enough to distinguish areas of high numbers of collisions and implement effective policy decisions and gives three reasons for this. First, there are potential inaccuracies in the collection of traffic collision points. Second, in urban areas it can be difficult to visually identify areas of high collision density. Third, by only considering points on a map there is a disregard for spatial or statistical patterns. Anderson suggests that looking at areas of high collision density, or hotspots, provides more robust analysis and better decisions. Anderson further states that "identifying accident hotspots and appending value added data to understand the processes occurring in these hotspots is important for the appropriate allocation of resources for safety improvements” (p. 359).

\subsection{Determining Hotspots}

Brimicombe (2005) states that the focus of the analysis of point data like traffic collisions is defining hotspots or blackspots where spatial clustering exists. If the focus is determining hotspots, then the first question should be "what is a hotspot?" There is no universally accepted definition of a traffic collision hotspot (Anderson 2006, 2009). Eck, Chainey, Cameron, Leitner, and Wilson (2005, p.2) indicate that while there is no common definition of the term hotspot, there is a common understanding that a hotspot is "an area that has a greater than average” number of events. Anderson $(2006,2009)$ and Brimicombe (2005) agree that a hotspot is generally defined as an area of high traffic collision density. Brimicombe then states that hotspots are areas where spatial clustering exists. Anderson (2009) also goes on to assert that hotspots "expose concentrations and therefore suggest spatial dependence between individual occurrences” (p. 360). She also points out that hotspots are not uniform in shape or size.

Grubesic and Murray (2001) note that there are a wide variety of methods to determine hotspots. They classify hotspot detection methods in five classes: visual interpretation, choropleth mapping, grid cell analysis, spatial autocorrelation, and cluster analysis. With a wide array of methods available, knowing which method to use would be helpful. They note that "although there are a variety of methods for detecting hotspots ... no single approach was found to be superior to others” (p. 1). Anderson (2006) also states that there is no universally accepted best method for determining hotspots of traffic collisions. Anderson points out that the best method to use varies depending on the data and the goal of the analysis.

While there is no superior method or universally accepted best method for determining hotspots, kernel density estimation (KDE) stands out amongst the literature as the most promising spatial tool for exploring the geography of point patterns (Sabel, Kingham, Nicholson, and Bartie, 2005). Chainey, Tompson, and Uhlig (2008) also suggest that KDE is the most suitable spatial analysis technique for visualizing event data. KDE has been used for hotspot detection by crime analysts and is now being used for traffic collision analysis. "There are many advantages of kernel density estimation (KDE) as opposed to statistical hotspot and clustering techniques such as K-means” 
(Anderson, 2009, p.360). According to Anderson, the main advantage of KDE is that is that it shows the spread of risk factor. Anderson explains that the risk associated with a collision isn't limited to a single point but is spread out around the incident and KDE shows this spread of risk. A second advantage is that by using KDE, "an arbitrary spatial unit of analysis can be defined and be homogeneous for the whole area which makes comparison and ultimately a taxonomy possible” (p. 360). A third advantage is that KDE "is able to quickly and visually identify hotspots from large datasets and therefore provide a statistical and aesthetically satisfactory outcome" (Anderson, 2006, p. 61). One more advantage pointed out by Williamson, McLafferty, McGuire, Mollenkopf, and Goldsmith (1999) is that KDE can clearly show complex spatial patterns in a smooth raster format. Both Williamson et al. and Anderson (2006) also note that due to the fixed cell size produced by KDE, it can be used to create new datasets and it can be combined with other datasets. All of these advantages provide a good reason to use KDE for determining hotspots.

When using KDE, there are several parameters that need to be set by the user. Bandwidth or search radius and cell size are two parameters that affect the outcome of the KDE (Anderson 2009, Brimicombe 2005). McCullagh (2006) and Wolff and Asche (2009) go on to state that both bandwidth and cell size selection are critical to a successful result. Anderson (2009) and Brimicombe (2005) note that the process of deciding bandwidth and grid cell size is somewhat subjective. This gives the analyst using KDE a certain amount of flexibility. Williamson et al. (1999) imply that this flexibility is both good and bad. It is good because it gives power to the analyst. Eck et al. (2005) also thinks this flexibility is good because it makes the analyst think about the data being used and select parameters that are appropriate for the data and the scale of the analysis. Williamson et al. (1999) also note that the flexibility can be bad if the analyst selects inappropriate parameters which can create misinformative maps. Brimicombe (2005) suggests that "best practice would suggest a form of sensitivity analysis to identify optimum parameter values" (p. 3). The bandwidth or search radius parameter for KDE is the distance the kernel looks around each cell to find points. Anderson (2009) states that "arguably the most important criterion for determining the most appropriate density surface is the bandwidth” (p. 361). Williamson et al. (1999) agree that the selection of bandwidth is a critical issue in KDE. The importance of selecting an appropriate bandwidth can be a problem since there are no hard and fast rules for determining bandwidth (Williamson et al. 1999). Wolff \& Asche (2009) also note that "bandwidth size selection is often more of an art than a science" (p. 148).

Fortunately there are many different suggestions for ways to determine an appropriate bandwidth. Williamson et al. (1999) discuss a few available methods. One method is to use a division of the shortest side of the extent of the point features. This is usually $1 / 30^{\text {th }}$ of the shortest side of the extent. Another method determines the bandwidth based on the number of points in the dataset, as well as the extent of the data. Williamson et al. recommend a method called k-means nearest neighbor distance. This method uses the average of the distances between each point and its $\mathrm{k}^{\text {th }}$ nearest neighbor. Williamson et al. state that this method is best because it takes into account the distribution of the points and not just the number of points or size of the extent. Downs and Horner (2007) discuss another available method of using least squares cross validation for determining bandwidth. They state that while this method is often used, it 
is problematic. This method is not appropriate when the sample size is small, when points are spatially or serially autocorrelated, or when the point pattern is linear or contains a large amount of empty space.

The other important parameter to determine for KDE is grid cell size. KDE places a raster grid over the point data and then uses a kernel to calculate each grid cell value. Like bandwidth, there are no hard and fast rules for selecting grid cell size and it is often subjective. Anderson (2009) recommends using a grid cells size that is half of the size of the bandwidth. Steenberghen et al. (2004) recommend using a grid cell size that is smaller than the length of street segments between intersections but large enough to still show traffic collision concentrations. Eck et al. (2005) recommend a method for determining grid cell size that is related to the extent of the crime point data. They recommend using the smaller side of the extent divided by 150 .

After the parameters have been determined and KDE has been performed, a continuous surface of densities is output. Since KDE results in a continuous surface, discrete hotspot boundaries still need to be defined (Wolff \& Asche, 2009). Anderson (2009) notes that "to be part of a hotspot a grid cell has to have an accident density level which is over a specified threshold" (p. 361). There are various methods for determining this threshold. Williamson et al. (1999) note that contours can be created from the KDE surface. A specific contour interval can then be chosen as the boundary for hotspots. Wolff and Asche (2009) present a method using neighborhood statistics. A 7x7 kernel is used to compute the standard deviation values for each cell. A cutoff value is then determined for the standard deviation values visually. The cutoff value is used to reclassify the raster and it is converted to polygon data. Another approach to determine hotspot boundaries is robust normalization. Brimicombe (2005) recommends this method. Robust normalization gives the median grid cell value a value of 0 , the lower quartile a value of -1 , and the upper quartile a value of +1 . This is applied to the density estimate interpolations. Hotspots are determined as cells with a value of greater than or equal to +3 . Eck et al. (2005) suggest using a multiple of the grid cells mean as the threshold value. They note that this method is simple, easy to implement, and consistent. A final method is suggested by Chainey, Tompson, and Uhlig (2008). They suggest using the quantile classification method. They used the quantile method with five classes, also known as quintiles. The highest quintile is the threshold value for hotspots.

After hotspots have been determined, it is important to add attribute data to the hotspot data. According to Anderson (2009), analysis should not be done on individual accidents but on hotspots that group accidents together that share a common nearby spatial location, implying a common causal factor. This grouping requires attribute data. Adding attribute data helps engineers determine any road or traffic conditions that can be changed to reduce traffic accidents (Lang, 1999). Lang also notes that important attributes for engineers to look at include accident cause, time of day, type of vehicles involved, type of collision, weather condition and severity.

\subsection{Summary}

The previous sections examined the broad use of GIS in the transportation industry and specifically its use in analyzing traffic collisions. The importance of analyzing traffic collisions and looking at collision hotspots was discussed. Various methods for determining hotspots were discussed and the advantages of using the kernel density 
estimation (KDE) method were considered in particular. The importance of selecting appropriate parameters for $\mathrm{KDE}$ was discussed, as well as the many methods available to do so. Finally, the available methods for determining a threshold value and the importance of adding attribute data to the hotspots were discussed. The next section reviews the design of the project based on the requirements of the client. 



\section{Chapter 3 - Systems Analysis and Design}

The client, the City of Redlands, recognizes the importance of implementing traffic collision reduction measures. This chapter focuses on the client's objectives of creating tools to help it effectively determine where traffic collision reduction measures are needed most and where such measures need to be implemented. The problem addressed by this project is examined first. The requirements of the project are then considered. Next, the design of the system that was implemented is discussed. Finally, the original plan for the successful completion of the project is reviewed.

\subsection{Problem Statement}

Traffic accidents are a common problem in any transportation network, and are compounded by the fact that traffic accidents often result in injury or the loss of life. The reduction of traffic collisions is a goal of transportation divisions at all levels of government. The City of Redlands wanted to determine where high numbers of traffic accidents are occurring within the city. Determining these areas will allow the city to concentrate on the areas of highest risk for traffic accident reduction. The city also needed to find any patterns or common attributes among the areas of high traffic accidents. Knowing any common attributes or patterns in these areas could help them understand why certain types of traffic accidents are occurring at certain locations.

\subsection{Requirements Analysis}

The analysis of the requirements for the project was broken down into two categories: functional and non-functional requirements. Functional requirements are the specific user needs and include what the system will answer or output. Non-functional requirements are the specifications on how the system should perform and are divided into technical, operational, and transitional requirements.

\subsubsection{Functional Requirements}

The functional requirements for this project were analyzed to determine how the system would function according to the user's needs (Table 1). The first functional requirement was that the data used in the analysis be point data representing traffic collisions. The client's traffic collision data was stored as point data and the analysis performed for the project had to be able to use this data directly as input. The second functional requirement was that the system must generate traffic collision hotspots. The final output of the analysis would be traffic collision hotspots represented as polygons. These hotspots would be attributed with the collision point data that was input into the analysis. The third functional requirement was that the system provide an application to examine the hotspots. This application would also be able to look at the attributes that were assigned to each hotspot. 
Table 1. Functional Requirements

\begin{tabular}{|l|l|}
\hline Function & Description \\
\hline \hline $\begin{array}{l}\text { The system will use traffic } \\
\text { collision point data as input }\end{array}$ & $\begin{array}{l}\text { The client has traffic collision data recorded as } \\
\text { points and the system needs to work with only this } \\
\text { input }\end{array}$ \\
\hline $\begin{array}{l}\text { The system will generate traffic } \\
\text { collision hotspots }\end{array}$ & $\begin{array}{l}\text { Based on the input, the system will generate an } \\
\text { attributed hotspot output }\end{array}$ \\
\hline $\begin{array}{l}\text { The system will provide an } \\
\text { application to examine the } \\
\text { hotspots and their attributes }\end{array}$ & $\begin{array}{l}\text { The client needs an application to be able to look at } \\
\text { the resulting hotspots and the attributes associated } \\
\text { with them }\end{array}$ \\
\hline
\end{tabular}

\subsubsection{Non-Functional Requirements}

This project needed be able to determine traffic collision hotspots and provide an application to easily examine the derived hotspots. The non-functional requirements (Table 2) discussed below focus on the ease of use of the developed system. They also look at the need of integration with the client's system and the need for simplicity of the system. The non-functional requirements were broken into technical, operational, and transitional requirements. 
Table 2. Non-Functional Requirements

\begin{tabular}{|l|l|}
\hline Function & Description \\
\hline \hline Technical & \\
\hline Software & ArcGIS Desktop and ArcGIS Server \\
\hline Computing Environment & $\begin{array}{l}\text { Desktop or laptop computer connected to client's } \\
\text { network }\end{array}$ \\
\hline Application GUI & Simple and easy to use \\
\hline Application Format & Readily available to client without additional cost \\
\hline Operational & \\
\hline User Expertise & Usable by someone with little to no GIS expertise \\
\hline Application Updates & Application can be updated by client if desired \\
\hline Transitional & \\
\hline Data & In ESRI format \\
\hline Deployment & Transitional errors will be fixed upon deployment \\
\hline Documentation and Training & $\begin{array}{l}\text { Documentation provided on analysis and } \\
\text { application } \\
\text { Little to no training on application use }\end{array}$ \\
\hline
\end{tabular}

\subsubsection{Technical Requirements}

The first technical requirement for the project was that it needed to use ESRI software, specifically ArcGIS Desktop and ArcGIS Server. These are the GIS products that the client already owns and it did not want to invest any more resources in GIS software for this project. Using these products also benefited the client in that adding any new software to its system would increase the complexity of the system and make maintenance more difficult.

The computing environment needed to be the same as the client's current computing environment. In order to use the application developed for this project, the user only needs a desktop or laptop computer. The user will also need to be connected to the internet to access the application.

The graphical user interface of the application needed to be simple and easy to use. The main reason for this was that the application was made available to city personnel, many of whom have no experience using GIS software. The graphical user interface needed to be simple and easy enough to use so that anyone with a basic understanding of using internet applications could use it. This also means that any menus involved in the application needed to be simple.

A fourth technical requirement was that the software used to develop the application and the final format of the application needed to be relatively common. The final deployed version of the application needed to be in a common format. If the 
application required any additional software then it needed to be software that is common and already installed on most of the client's computers.

\subsubsection{Operational Requirements}

The first operational requirement was that the application needed to be user friendly since the users of the application may have little to no GIS experience. These users should be able to easily use the application despite their lack of GIS software experience. The tools and buttons should be easy to find and use. The functionality of the tools in the application should also be self explanatory.

The second operational requirement was that the application developed for this project needed to be able to be updated by the client. Using the methodology from this project, the client will be able to determine traffic collision hotspots using collision data from time periods other than that used by this project. The web application needed to be easily updated with this hotspot data.

\subsubsection{Transitional Requirements}

The first transitional requirement included transferring data to the client's system. The client uses ESRI software, specifically ArcGIS Desktop and ArcGIS Server. The data provided by this project needed to be in a format that is usable by this software without any conversion.

The application developed by this project was not developed on the client's system. This could have caused problems when the application was transferred to the client's server. The application code was edited to fix any broken file paths. Any other errors in the code created by the transition were also fixed before deployment.

Another transitional requirement was documentation and training on the application. No training on the use of the application was given because the application is simple and intuitive. However, some training on the coding and deployment of the application was given. This was done in case the client wants to change or update the application in the future. Documentation was also provided in the form of written documentation and thorough commenting of the application code.

\subsection{System Design}

This section discusses the hardware and software that was used in the project based on the client's needs. The technical solutions are also determined based on the objectives of this project, the needs of the client, and the requirements previously outlined. The concept for the system is that it required an input of traffic collision points. The development of the database for this data is discussed in Chapter 4. Using this input, the system determined the location of traffic collision hotspots. An application was then provided to examine these hotspots and their attributes.

The environment used for the determination of hotspots is ESRI's ModelBuilder. This was chosen because it provides an automated framework for running the various tools provided in ESRI's software. This automation is beneficial if the client chooses to use the methodology from this project on other datasets. ModelBuilder was also ideal because it documents the processes involved in the analysis and allows for repeatable 
results. This decision also supports to client's needs as it does not require any additional software.

The application for exploring the hotspots was developed as a web application mainly because it is a format that is readily available to everyone at the client's organization and can be used by anyone on the client's network who has an internet browser. No additional software will be required for the use of the application. Another benefit of using a web application is that the graphical user interface was designed to be relatively simple and user friendly. This was done to make the application usable to those without any GIS experience.

The web application was developed using Adobe Flex and the ArcGIS Application Programming Interface (API) for Flex. Flex was chosen because applications developed in Flex can run on all major browsers. Flex web applications need only Adobe Flash Player to run, which reportedly is installed on over $98 \%$ of internet connected computers (Adobe, 2009). This requires no additional software for the client and makes it available to all the users on their network. Flex was also chosen because ESRI has released an API for Flex that allows data in ESRI formats to be used easily in web mapping applications. This was beneficial since the data from this project was in an ESRI file geodatabase. Flex also has components for creating charts and tables from data which were very helpful in the development of the web application.

The system architecture used for this project was limited to what was currently available to the client. The web application required a server with ArcGIS Server software installed on it. The client already has this as a part of their system, so nothing further needed to be added for the web application. If the client would like to run the model developed in this project they will need ESRI's ArcGIS Desktop software. ArcGIS Desktop has three license levels: ArcView, ArcEditor, and ArcInfo, with ArcInfo having the most functionality. Running the model requires an ArcView license and the Spatial Analyst extension. The client also already has this software and no further software is required.

\subsection{Project Plan}

This section outlines the original plan for completion of the project broken down by task. These tasks were determined as what was necessary to meet the goals and objectives of the project. The tasks are presented in a linear fashion, but some overlap between tasks

did occur. The follow chart (Figure 3.1) gives an outline of the tasks to be discussed. 


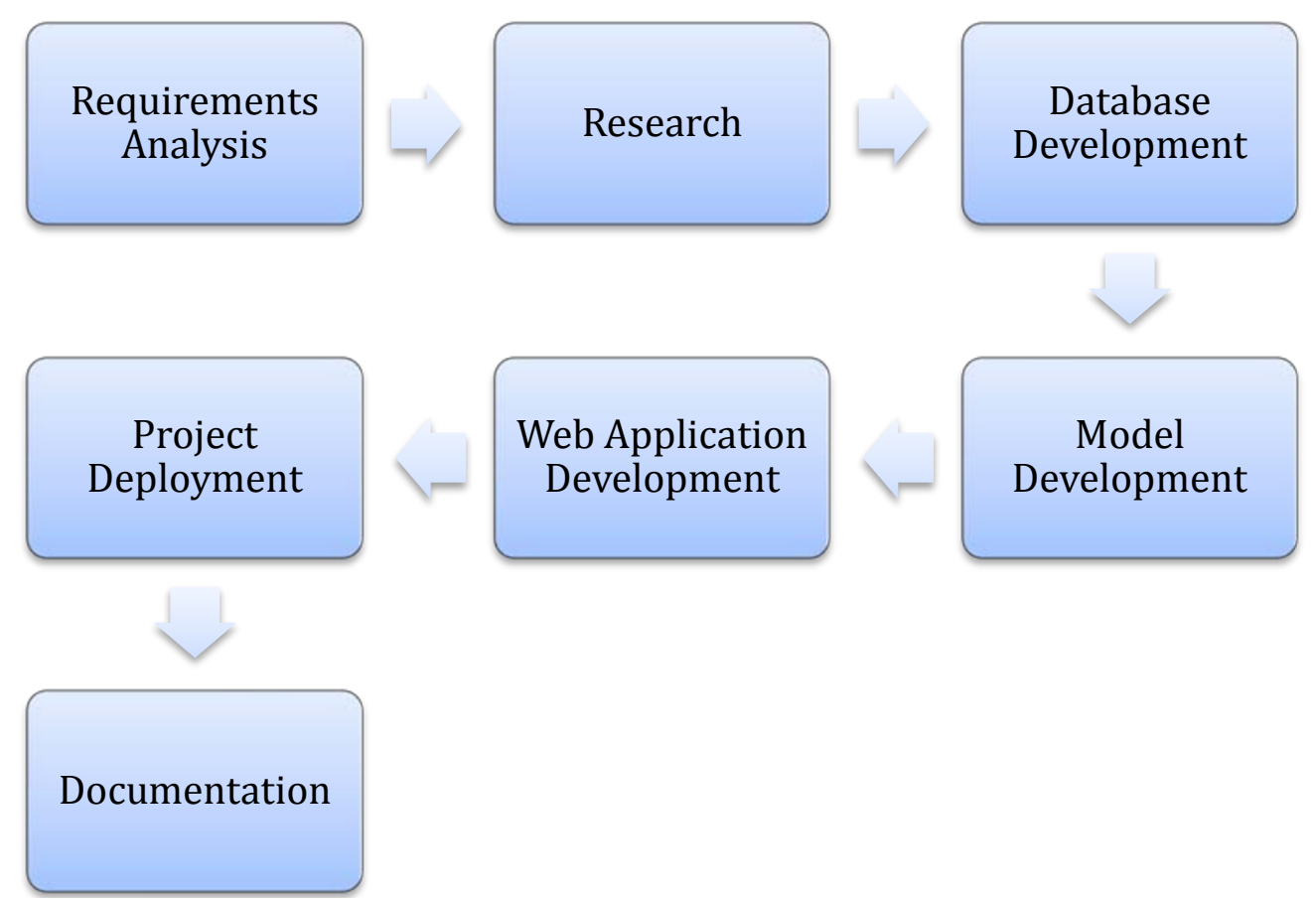

Figure 3.1 Project Plan Tasks

\subsubsection{Requirements Analysis}

The first task for this project was to perform a requirements analysis. This was accomplished through meetings with Phil Mielke, the point of contact for the project. The goal of these meetings was to determine the problem that the client wanted addressed. Once the problem was known, the goals and objectives of the project were laid out. These were later used to determine the success of the project. The scope of the project was also determined. A high level methodology was outlined as a possible solution to the client's problem. This methodology was used to determine the tasks that would need to be completed in the project, as well as the milestones and deliverables for the project.

\subsubsection{Research}

The second task for the project was performing the research necessary to complete the project. The goal of this task was to determine an appropriate methodology for addressing the client's problem. Materials regarding the use of GIS in the transportation industry were examined. Next, materials regarding the analysis of traffic collisions were looked at, specifically the analysis of traffic collision hotspots. The most difficult part of this task was determining the methodology to be used for determining the hotspots as numerous methods are available. The parameters required by the chosen methodology for determining hotspots were also looked into in this task. 


\subsubsection{Database Development}

The third task for the project was to develop a database for the project data. First, a conceptual design of the database was completed. This design determined which data would be used in the project. The important attributes for the data were also determined at this stage. With the conceptual design complete, a logical model of the database was developed. The database was created as an ESRI file geodatabase and the data from the client were then loaded into it. The data also required some cleaning to remove bad records and to normalize some attribute values. This is discussed in Chapter 4.

\subsubsection{Model Development}

The fourth task for the project was developing a model to determine traffic collision hotspots and adding attribute data to the hotspots. Based on the research done in the second task, a methodology for determining hotspots was laid out. Once the methodology to be used was set, determining how to implement the methodology in ModelBuilder was the next task. There were some challenges in figuring out how to accomplish certain tasks using the available tools in the ArcGIS Desktop software. After the model was built, a sensitivity analysis was done to determine the best values for the parameters of the model. The model was run using various values for the cell size and bandwidth parameters. The results were compared to determine the best values for the parameters. Once the parameters were set, the model was run and the final results for the hotspots were saved into the database.

\subsubsection{Web Application Development}

The fifth task was developing the web application for examining the hotspot data generated by the model. The data from the hotspot model was published for use by the web application using ArcGIS Server on the project machine. With the necessary data available, the web application could be designed and developed. The functionality and the layout of the application were determined. The layout and toolbars were then added to the application and the functionality of the application was coded. Testing of the functionality of the code was done throughout the process. Final testing was done to complete the application.

\subsubsection{Project Deployment}

The sixth task was deploying the web application and data onto the client's network. The database was moved over to the client's network and the data for the web application was published on the client's server using ArcGIS Server. The links to the data in the application code were then changed to point to the data on the client's server. At this point the web application was tested again to ensure that no errors were introduced with the migration of the data. After testing was completed, the application was compiled and moved to the client's server. The application was tested once more after it was deployed on the client's network. The client was given a small amount of training on the model and application. 


\subsubsection{Documentation}

The final task was documenting the project. A draft of the project report was completed, followed by editing. After various edits were applied, a final version was submitted to conclude the project.

\subsection{Summary}

The sections discussed in this chapter looked at the problem the client needed solved by this project. After examining the problem, the user requirements were considered. These requirements included both functional and non-functional requirements. The system for this project was then designed based on the user requirements, as well as the research previously completed. With the system designed, the project plan was laid out, including the specific tasks that were completed. 


\section{Chapter 4 - Database Design}

Once the system for this project was designed, the next step was to develop a project database to hold the input data and act as a storage place for the model and its results. This chapter focuses on the design of the database. First, the conceptual design of the database is discussed. The logical data model as an ESRI File Geodatabase is then reviewed. The features classes included in the logical model are detailed, and the data sources are then discussed. Finally, the loading of the data into the database and the cleaning of the data is examined.

\subsection{Conceptual Data Model}

Conceptual data models define the data that are to be included, the characteristics of the data, and the relationships between the data. The starting point for the conceptual model design was the functional requirements that were previously discussed in Chapter Two. One of the functional requirements was that the analysis model developed in this project had to work with traffic collision point data as the only data input. This was used to determine that the main dataset in the data model would be for traffic collisions. Datasets other than the traffic collision dataset were kept to a minimum as they were only used as reference data. This also helped to reduce the complexity of the data model.

The conceptual data model (Figure 4.1) includes three datasets. The main dataset is the collision dataset. This was determined from the functional requirements as previously stated. The collision dataset has ten attributes: collision report number, date of the collision, time of the collision, address or intersection of the collision, severity of the collision, type of collision, lighting conditions at the time of collision, weather conditions at the time of collision, number of people injured in the collision, and number of people killed in the collision. These ten attributes were determined based on the research performed and with input from the client. Two other datasets were also included in the conceptual data model: traffic signals and stop signs. Each of these datasets includes a unique ID attribute which is included since it is required by the database management software. No other attributes were included since the traffic signal and stop sign data were only going to be used as reference data in the final web application. It is also important to note that there are no relationships between the datasets in the model. 


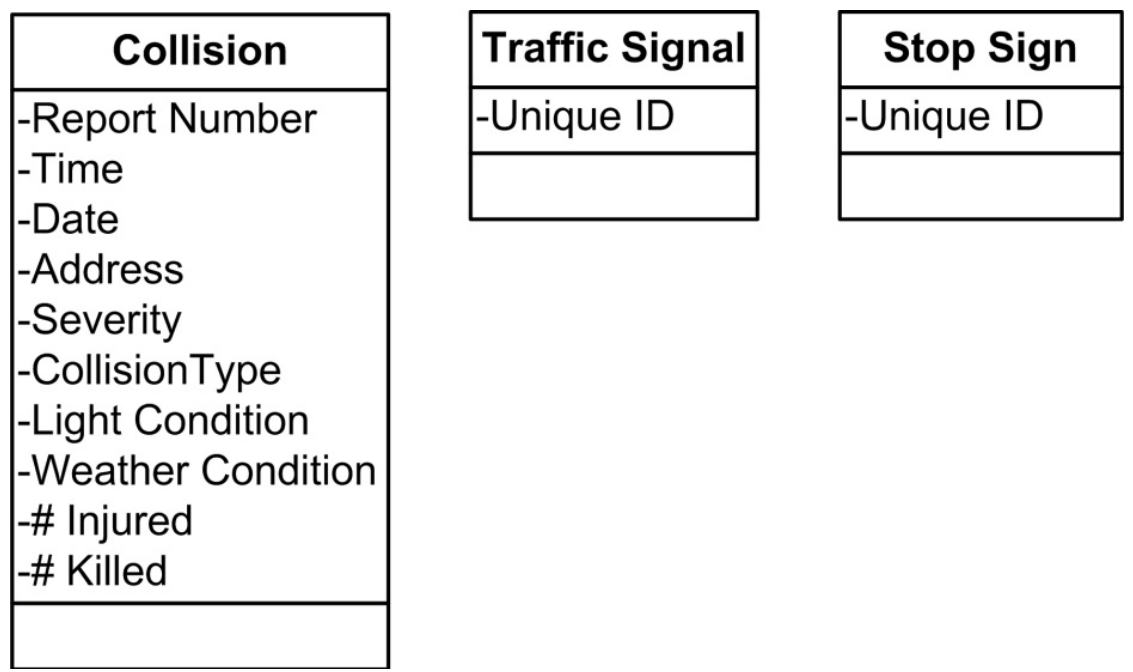

Figure 4.1 Conceptual Data Model

\subsection{Logical Data Model}

After the conceptual data model had been completed, a logical data model was derived from it and was designed as an ESRI file geodatabase. The feature classes, their attributes, and their relationships are laid out in the logical data model. The following section discusses the design of the logical data model for this project.

\subsubsection{Collision Hotspot File Geodatabase}

The logical data model was implemented as a file geodatabase. This format was chosen because of its simplicity of use and compatibility with the software used in this project. The feature classes in a file geodatabase can easily be used in ArcMap and ModelBuilder and published for use by a web application using ArcGIS Server. The file geodatabase was chosen for this project because the model developed in ModelBuilder and the output of the model can be stored in the file geodatabase. The file geodatabase has adequate storage capacity for the needs of the project.

When the file geodatabase was created the map projection was determined, as well. The NAD_1983_StatePlane_California_V_FIPS_0405_Feet projection was used. This projection is a Lambert Conformal Conic projection that uses the North American 1983 datum and the California state plane coordinate system with feet as the unit of measure. This is the projection used by the City of Redlands. The source data was in this projection and all output needed to be in this same projection. It is a conformal projection and preserves angles so the shapes of features look like they should. This projection is appropriate for this project since the analyses are exploratory and no areal measurements are being calculated. 


\subsubsection{Collision Feature Class}

The main feature class in the file geodatabase is the collision feature class (Figure 4.2). The logical model is very similar to what was laid out in the conceptual model, but two attribute fields, OBJECTID and SHAPE, were added by the software when the feature class was created. The rest of the attributes were taken directly from the conceptual model. The data types for the attributes were set when the feature class was created. The IncidentID, CollisionTime, Address, Severity, CollisionType, LightCondition, and WeatherCondition attributes were set as text data types. The CollisionDate attribute was set as a date data type and the NumInjured and NumKilled attributes were set as short integer data types.

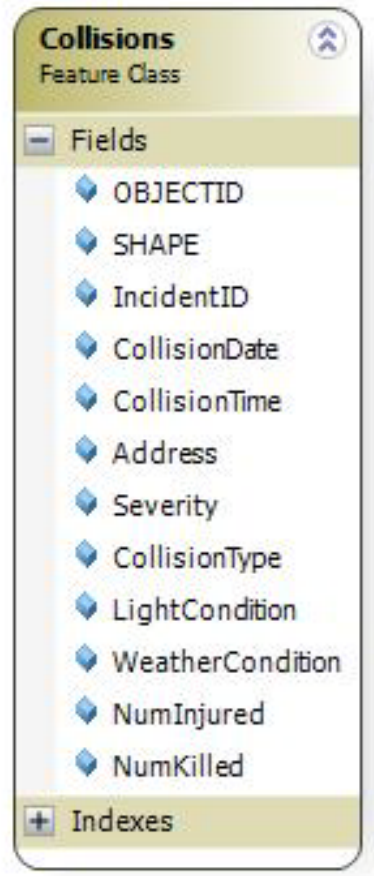

\section{Figure 4.2 Collision Feature Class}

One consideration when creating this feature class was the use of domains for some of the attributes. Domains place limits on the input for an attribute field, which can be useful when entering data in attribute fields that have limited numbers of acceptable values. The use of coded value domains was considered for this feature class but was decided against because once the data was loaded into the feature class it was not going to be edited, so the use of domains would not be helpful.

\subsubsection{Traffic Signal and Stop Sign Feature Classes}

Feature classes for traffic signals and stop signs were also created in the logical model (Figure 4.3). These feature classes were taken directly from the conceptual model, as well. Each of the feature classes has the OBJECTID and SHAPE attributes, created and managed by the software. No other attributes were added to these feature classes since 
they were only used as visual reference data in the final web application. These feature classes do not have any domains or relationships between them.
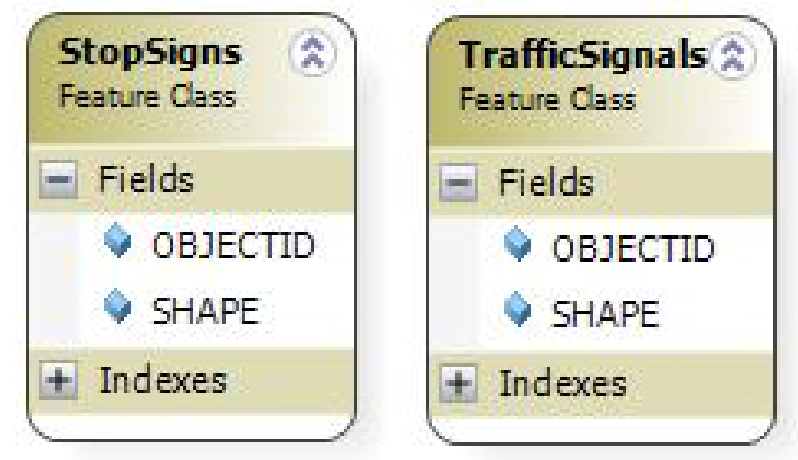

Figure 4.3 Traffic Signal and Stop Sign Feature Classes

\subsection{Data Sources}

All of the data that were put into the data model were provided by the City of Redlands. The collision data were provided in shapefile format and the traffic signal and stop sign data were provided in a file geodatabase format. All of the data were provided in the NAD_1983_StatePlane_California_V_FIPS_0405_Feet projection.

It should be noted that data from ArcGIS Online were used in the final web application. ArcGIS Online provides free and for-pay base map services. The free versions of the World Street Map and World Imagery were used by the web application in this project. These maps are created and maintained by ESRI and are provided in the GCS_WGS_1984 projection. When these base maps are used in a web application, any other data added to the web application needs to be converted to this projection.

\subsection{Data Scrubbing and Loading}

After the logical data model was created and the data were received from the client, the data were loaded into the database. This process was fairly straightforward since the data were being transferred between ESRI formats. The Simple Data Loader tool in ArcCatalog was used to load the data into the feature classes, which provided an easy interface for matching field names between the source and the target databases. This was useful as many of the field names in the collision feature class were different from the field names in the collision data provided by the City of Redlands (Figure 4.4). Another useful feature of the Simple Data Loader tool is that it allows for the data that are transferred to be defined by a query. This was used with the stop sign feature class since the stop sign data provided by the City of Redlands were part of a feature class containing all of the street signs in the city. A query was used to transfer only the stop sign features. 


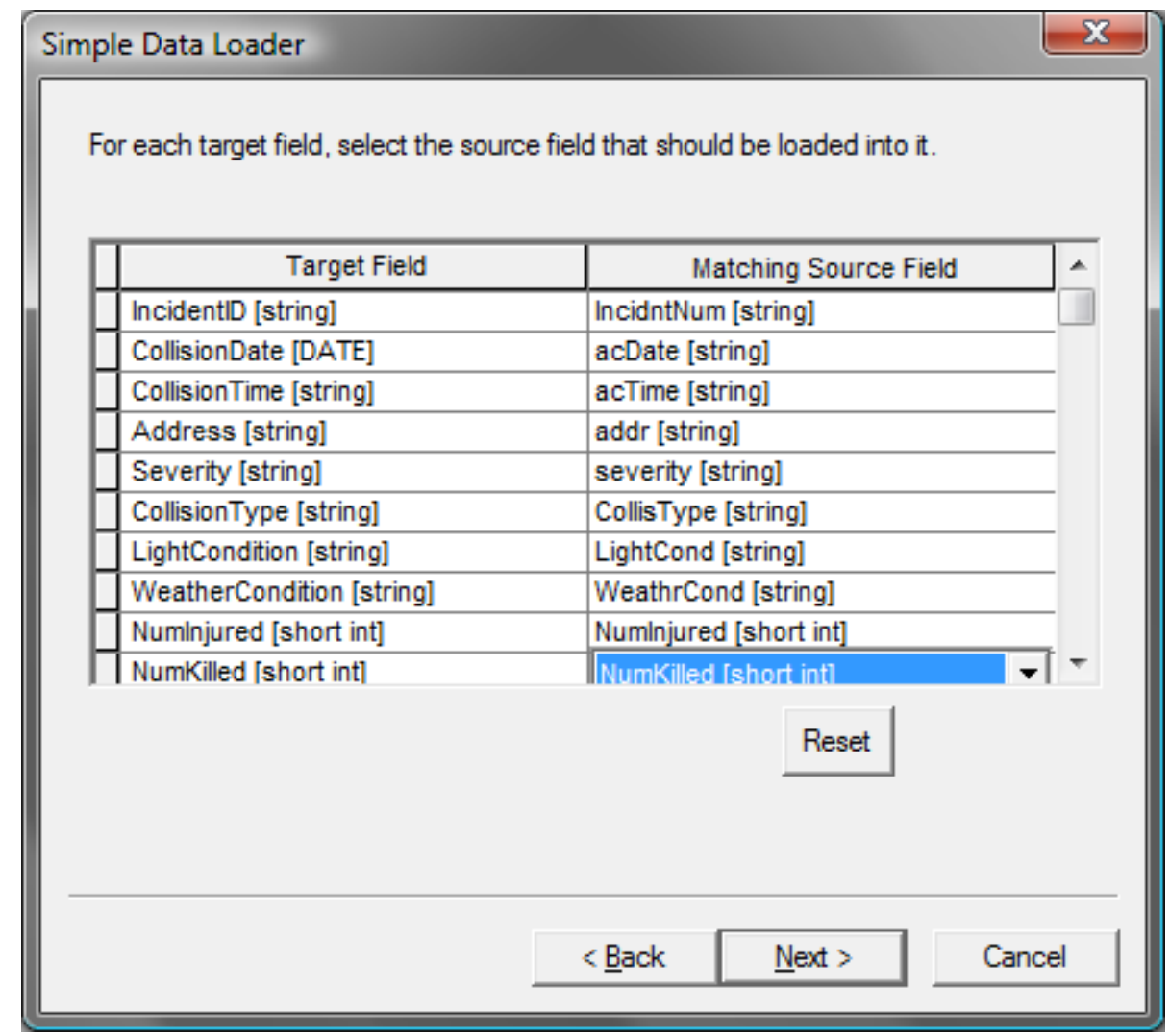

Figure 4.4 Collision Data Loading

After the data were loaded into the file geodatabase some scrubbing of the data was necessary. One issue was the lack of consistency in the case of the attributes in the collision feature class. Some of the records were in uppercase and some were in proper case. This created problems when querying the data since the same value is seen as different by the query if they are in different cases. This was a problem with the Address, Severity, CollisionType, LightCondition, and WeatherCondition attributes. This was addressed by using the Field Calculator in ArcMap which provides an interface for calculating the attribute values for a field in a feature class and allows Visual Basic functions to be used in the calculations. The StrConv function was used to recalculate the values for the Address, Severity, CollisionType, LightCondition, and WeatherCondition fields (Figure 4.5), as well as to convert all of the records to proper case, where the first letter of each word is upper case and the rest are lower case. Converting the values to proper case also helped increase readability of the text. The Field Calculator was also used on the CollisionTime field to add a colon between the hour and minute numbers, which was done to increase readability. 


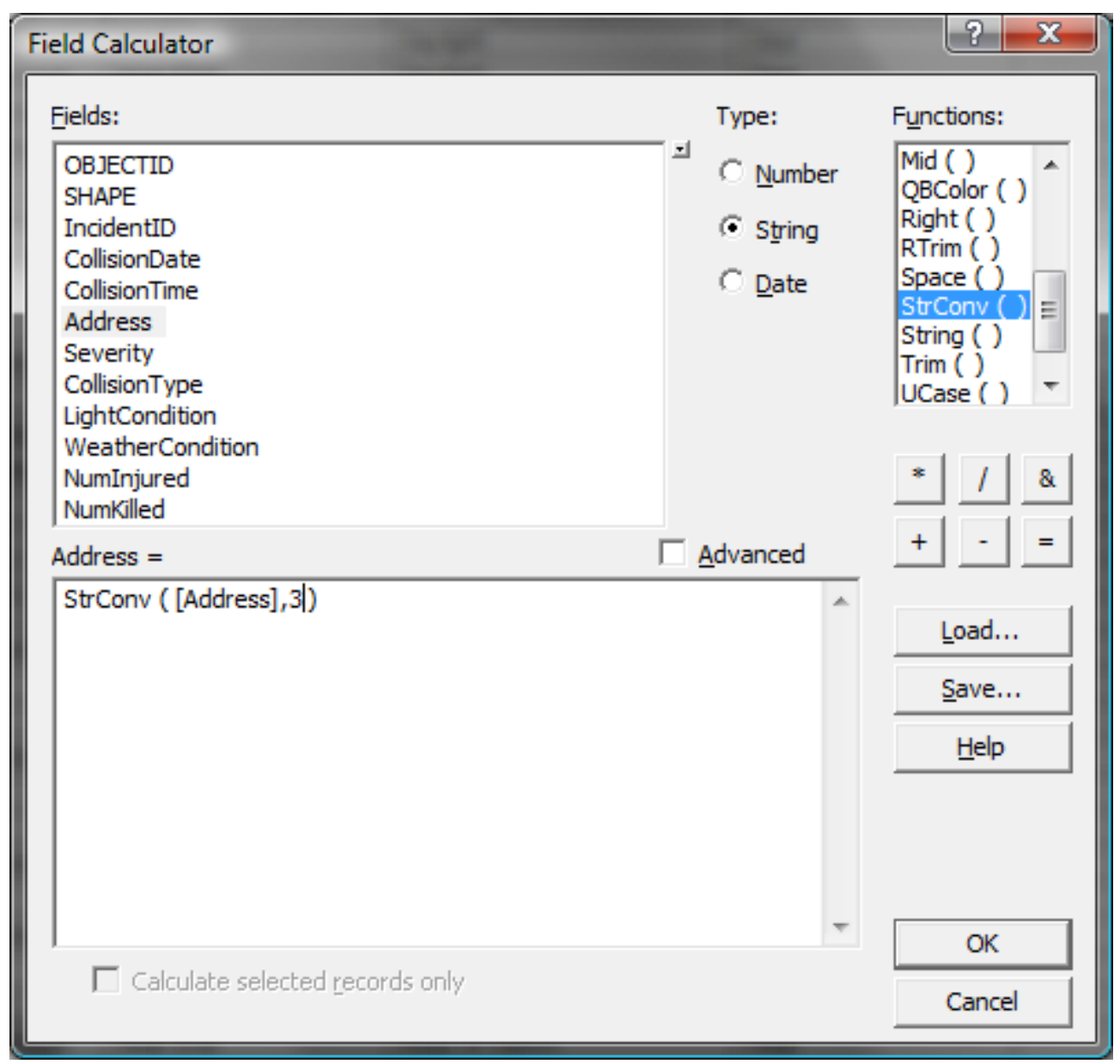

Figure 4.5 Calculation for Converting Fields to Proper Case

Another data scrubbing issue involved duplicate records in the collision feature class. It was noticed that some of the features in the collision feature class had a null geometry value. These features would cause an error when run through the analysis tools. After examining the records that were causing the error, it was determined that these records were duplicates. There were 112 duplicate records in the collision feature class. The Repair Geometry tool in ArcMap was used to remove the duplicate records. This reduced the total number of collision features from 2137 to 2025.

\subsection{Summary}

After reviewing the requirements laid out in the previous chapter, the conceptual data model was designed. This conceptual data model was used to create the logical data model. The logical data model was implemented as a file geodatabase. The data sources for this project were discussed and the data loading tasks were examined. With the data loaded into the file geodatabase, data scrubbing tasks were discussed. 


\section{Chapter 5 - Implementation}

Once the database was designed and the data was loaded and cleaned, the implementation of the hotspot detection model and the web application were completed. This chapter describes how the hotspot detection model and web application were implemented. The tools used in ModelBuilder and the determination of parameters for the tools are discussed. The programming methods implemented in the web applications are then examined.

\subsection{Hotspot Detection Model}

The first part of implementation was creating the hotspot detection model in ModelBuilder. Models need to be stored in a toolbox, so a toolbox was added to the project file geodatabase, allowing the model to be stored in the same location as the data. The hotspot detection model (Figure 5.1) was then created and saved in the toolbox. The model was broken down into three steps: kernel density estimation, reclassification, and adding collision attribute data. The following sections discuss the steps and tools used in the hotspot detection model and detail the values that were determined for these tools.

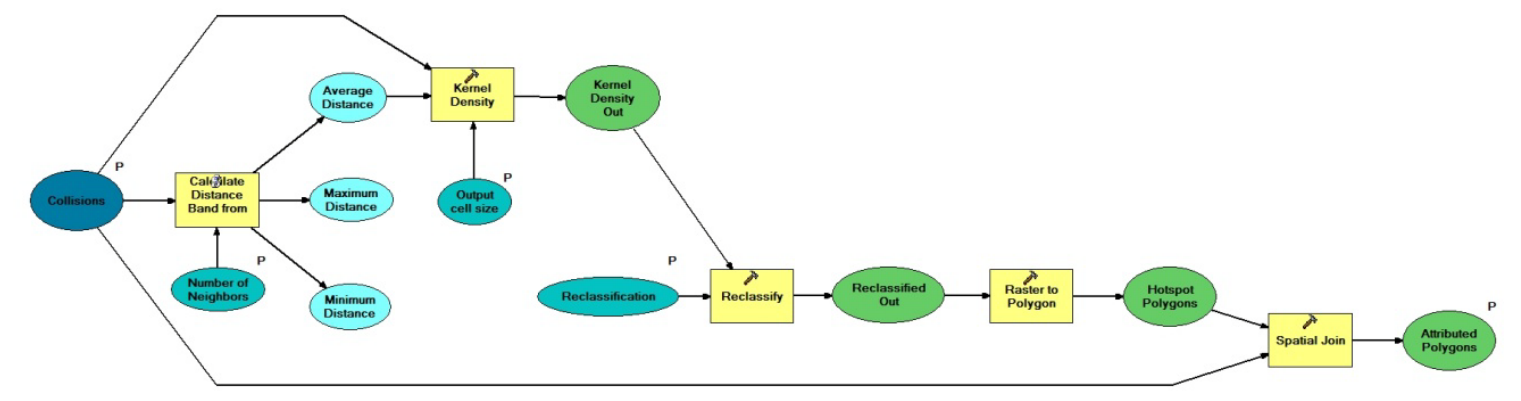

Figure 5.1 Complete Hotspot Detection Model

\subsubsection{Kernel Density Estimation}

The first step of the model was creating a density surface of the collisions using kernel density estimation (KDE). KDE was determined to be an appropriate method for determining traffic collision hotspots based on the literature review performed in Chapter Two. KDE works by creating a raster density surface over the extent of the data. First, a grid of square cells is placed over the extent of the point data. Then the density value for each cell is calculated using a kernel. The kernel looks for points within a certain distance, known as the bandwidth, from the cell. Points closer to the cell are given a higher weight in the density calculation than points further away from the cell. The final result is a smooth raster surface of densities. The size of the grid cells and the bandwidth distance greatly affect the output of $\mathrm{KDE}$, so these values were carefully determined based on guidelines from the literature review. 


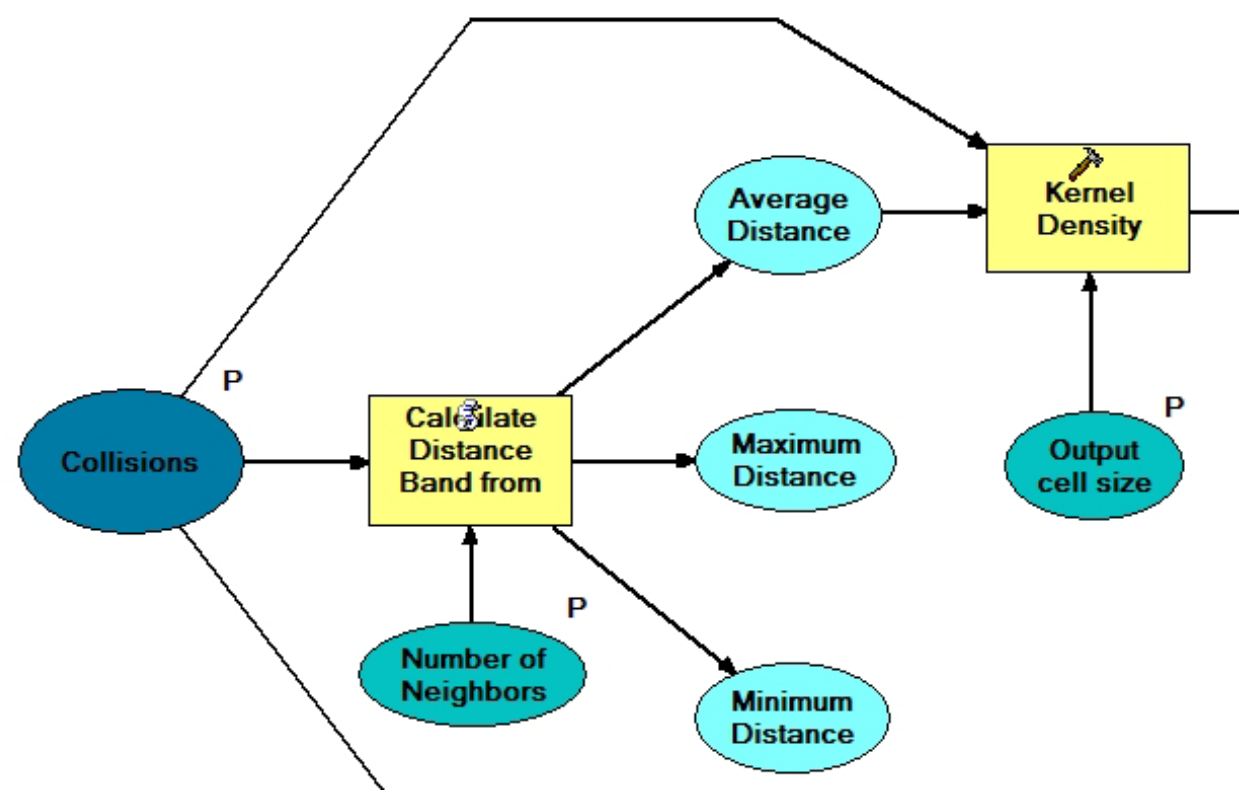

Figure 5.2 Kernel Density Estimation Step in the Model

The bandwidth is the distance that the kernel searches for points when determining the density value for each cell. While there are no hard and fast rules for determining the bandwidth, there are many guidelines. Calculations using the extent of the data or the number of points in the dataset have been suggested and were discussed in Chapter Two. The method used in this project is the average k-th nearest neighbor distance. This method was recommended by Williamson et al. (1999) since it considers the distribution of points in the data instead of just the extent or number of points.

Average k-th nearest neighbor distance looks at the distance between points in the dataset. The distance between each point and its k-th nearest neighbor is calculated and the distances are then averaged. This calculation was implemented in the ArcGIS software using the Calculate Distance Band from Neighbor Count tool in the Spatial Statistics toolbox. One parameter for the tool is whether Euclidean or Manhattan distance is to be used in the calculation. Euclidean distance is the straight line distance between points while Manhattan distance is the distance between points measured along axes at a right angle. Manhattan distance was used because it is closer to the distance between points when traveling on a road network. The order of neighbors to include in the calculation is also required as input. A sensitivity analysis was performed to determine the appropriate order of neighbors to use for the calculation, an approach suggested by Brimicombe (2005). Kernel density surfaces were created using the average first, third, and fifth nearest neighbor distances as bandwidths (Figure 5.3). Shorter bandwidths create density surfaces with smaller, spikier hotspots, and longer bandwidths create density surfaces with larger, more blended hotspots. The average third nearest neighbor distance was used in the model. This bandwidth provided a balance between numerous small hotspots and a few large hotspots. 


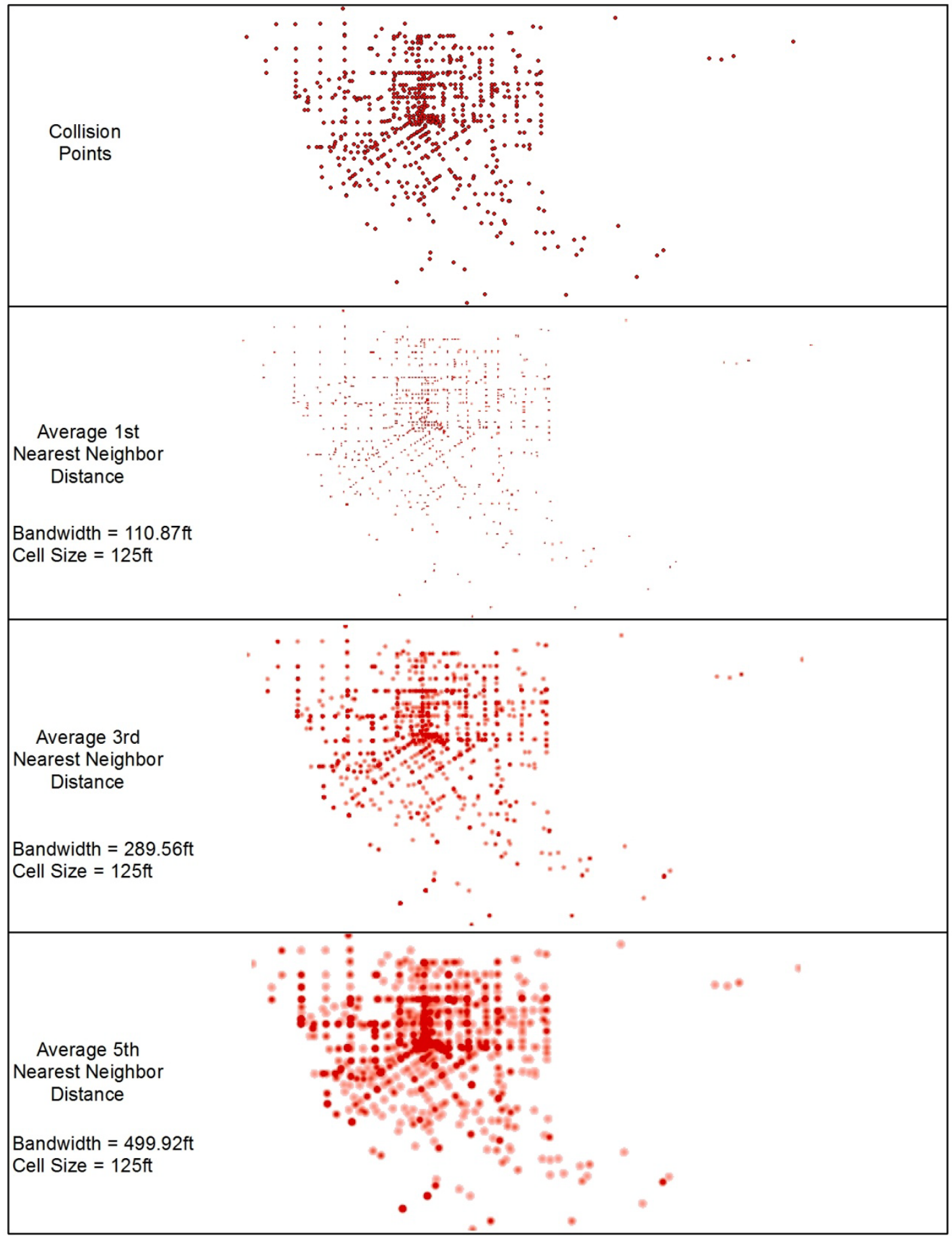

Figure 5.3 Bandwidth Selections for Kernel Density

The cell size for the kernel density was also determined based on a sensitivity analysis. Density surfaces were created using 50, 125, and 200 foot cell sizes (Figure 5.4). Smaller cell sizes create smoother density surfaces but larger file sizes, while larger cell sizes create rougher density surfaces but smaller file sizes. A cell size of 125 feet was 
used in the model for this project. This value was used because it fit both Anderson's (2009) recommendation that the cell size be approximately half the size of the bandwidth, and the recommendation of Steenberghen et al. (2004) that the cell size be smaller than the length of the street segments between intersections.

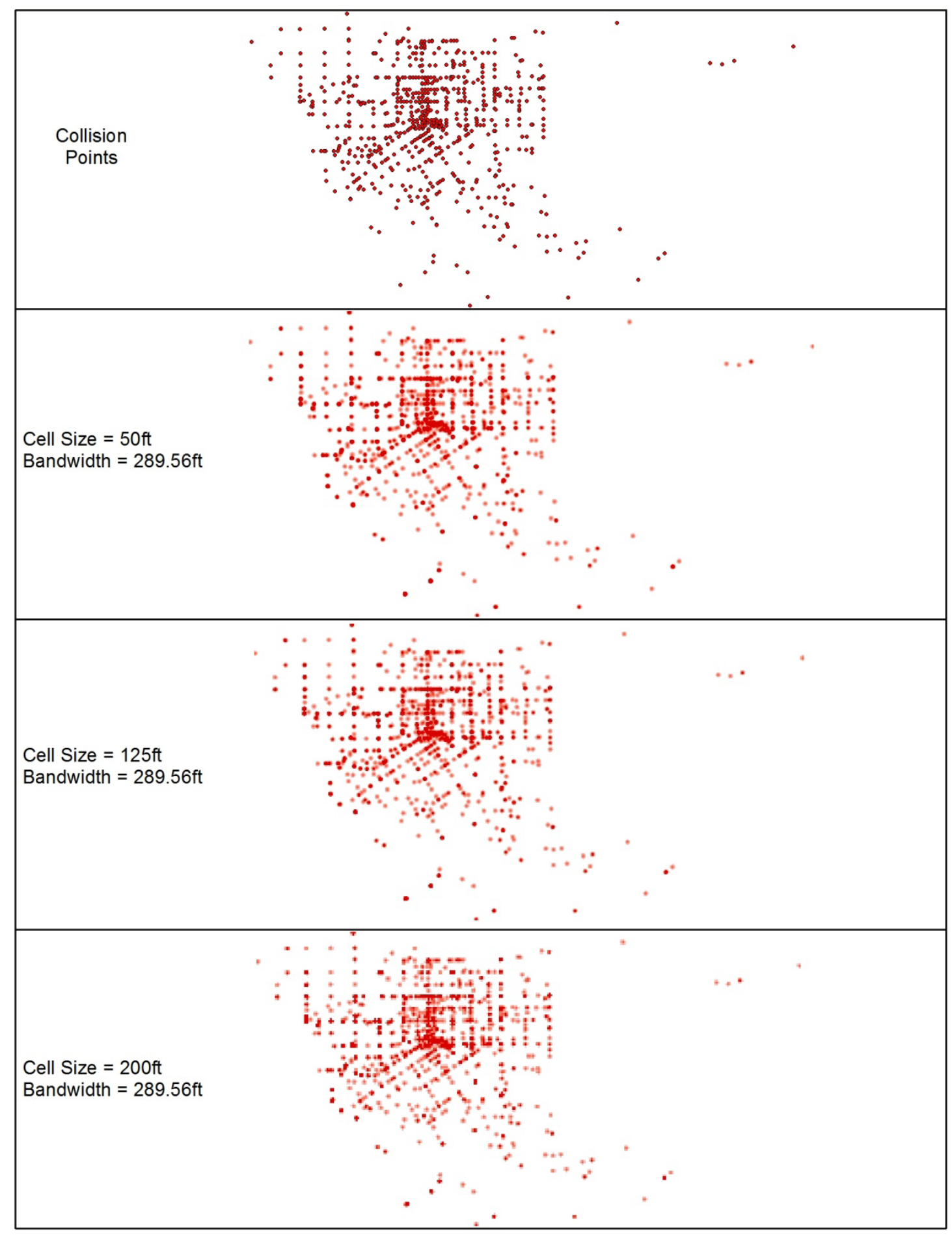

Figure 5.4 Cell Size Selections for Kernel Density 


\subsubsection{Reclassification}

Once the kernel density surface was created, the cell values needed to be reclassified in order to delineate the traffic collision hotspots. The density values were reclassified into one of two values: a value of one if it was a part of a hotspot and a no data value if it was not a part of a hotspot. This reclassification was done using the Reclassify tool in the Spatial Analyst toolbox. In order to determine if a cell was to be included in a hotspot, a cutoff value was determined. A cell's value had to be above the cutoff value to be included in a hotspot.

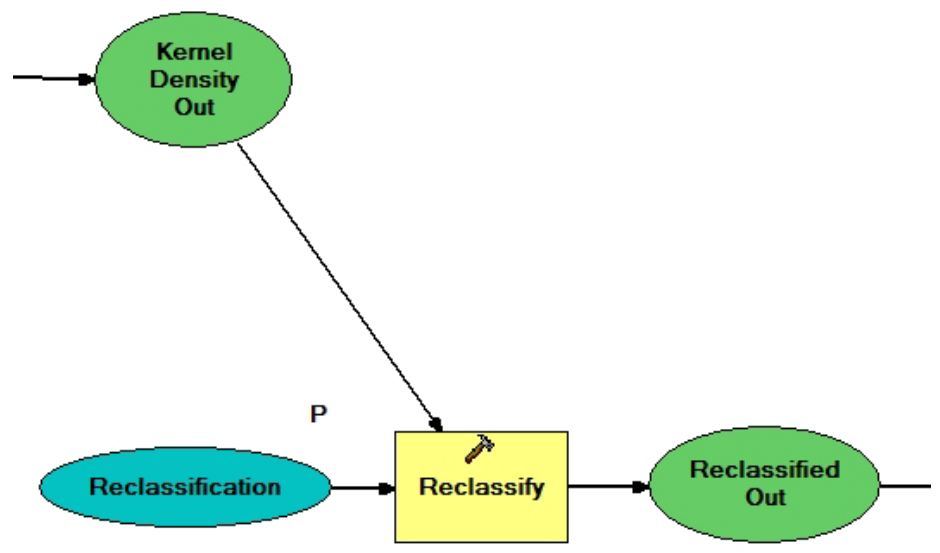

Figure 5.5 Reclassification Step in the Model

There are many different methods available for determining a cutoff value when reclassifying data. Three different methods were applied to the kernel density surface to determine which was most appropriate for this project (Figure 5.6). In each of these methods the cells with a value of zero were excluded from the calculations, as recommended by Eck et al. (2005), because a majority of the cell values in the raster were zero. This was caused by the kernel density estimation creating a raster surface that covers the entire extent of the study area, while traffic collisions only occur on the road network within the study area. Including the zero values in the mean and median calculations would greatly reduce the calculated cutoff value and result in any cell with a density value greater than zero being considered a hotspot.

The first method, suggested by Eck et al. (2005), used multiples of the mean cell value. The cutoff values for the classes are determined by multiples of the mean cell value. Cells with a value of five times the mean cell value or higher are considered to be a part of a hotspot. The second method used quantile classification, as suggested by Chainey, Tompson, and Uhlig (2008). Quantile classification was done using five classes. The highest value class, which contains the highest $20 \%$ of the cell values, was used as the cutoff value. The third method used was robust normalization, as suggested by Brimicombe (2005). Robust normalization uses the median and upper and lower quartile values in classification. The median cell value is given a value of zero. The upper quartile cell value is given a value of one. The range between the median and the upper quartile is 
used multiple times to get values of two and three. The cell value that is given a value of three is used as the cutoff value.

The method using multiples of the mean cell value was determined to be the most appropriate for this project since it produced the smallest hotspots, which were limited to a single intersection or road segment. The other methods produced hotspots which covered multiple intersections or road segments, making finding any commonalities within a hotspot more difficult. The mean cell value multiplied by five-2,556.189 points per square mile-was used as the cutoff value for the reclassification. 


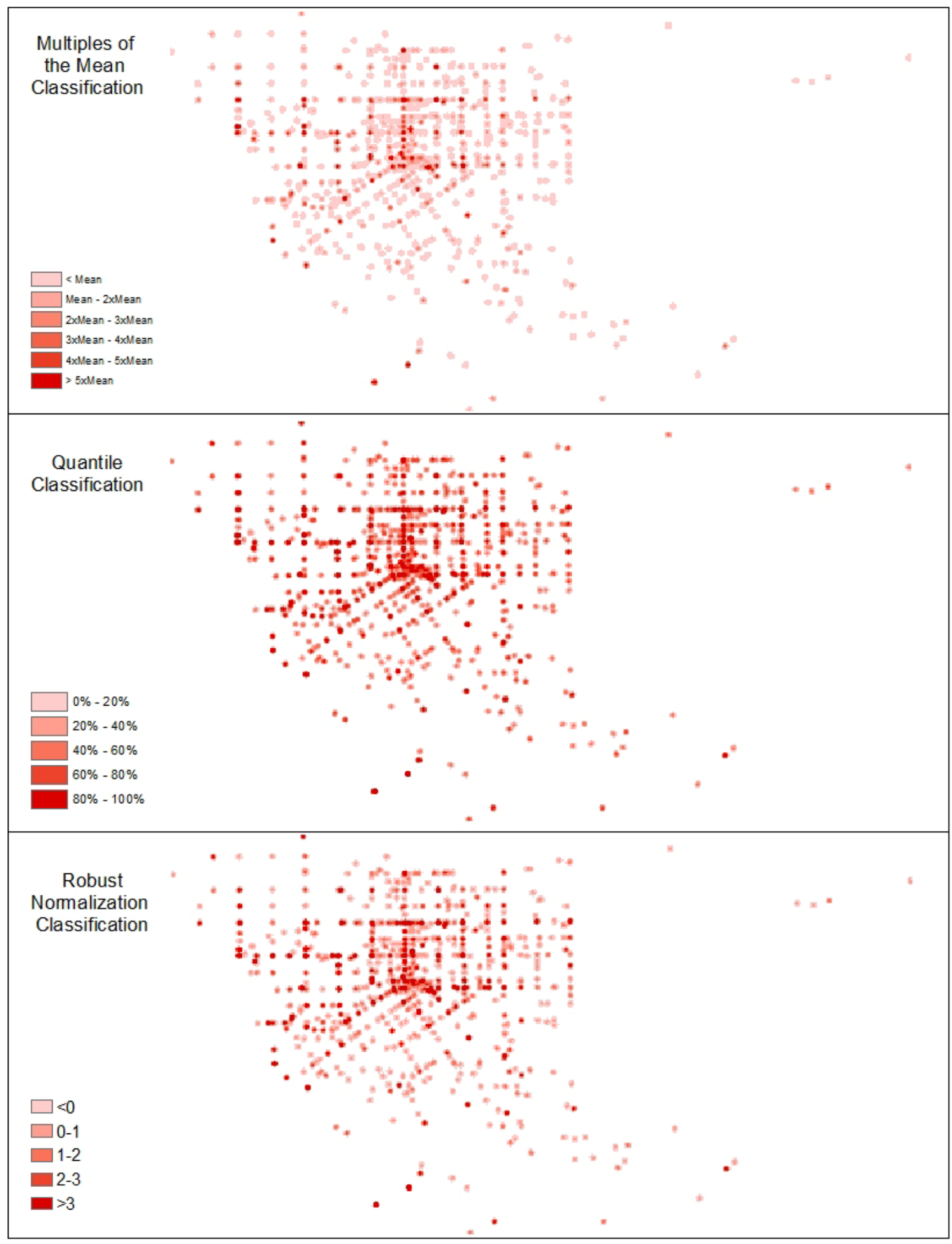

Figure 5.6 Reclassification Methods for Kernel Density 


\subsubsection{Adding Collision Attribute Data}

After the kernel density surface was reclassified, the hotspots were converted to polygons. This was done with the Raster to Polygon tool in the Conversion toolbox. The tool takes raster cells with the same value that also share a side and converts them into a polygon. Creating hotspot polygons was necessary for attribute data from the collisions to be added to the hotspots.

The final step in the hotspot detection model was adding attribute data to the hotspot polygons (Figure 5.7). This was done using the Spatial Join tool in the Analysis toolbox. The Spatial Join tool added three new attribute fields to the hotspot polygon feature class. The first was the Join_Count field, which contains a count of the number of collision points that are within each hotspot polygon. The other two added fields are the NumInjured and NumKilled fields. These fields contain the total number of people injured or killed in the traffic collisions that occurred within each hotspot polygon. The Join_Count was automatically created by the Spatial Join tool while the NumInjured and NumKilled fields were added based on user input. User defined fields need to have a merge rule assigned to them in the tool. The sum rule was used for both the NumInjured and NumKilled fields.

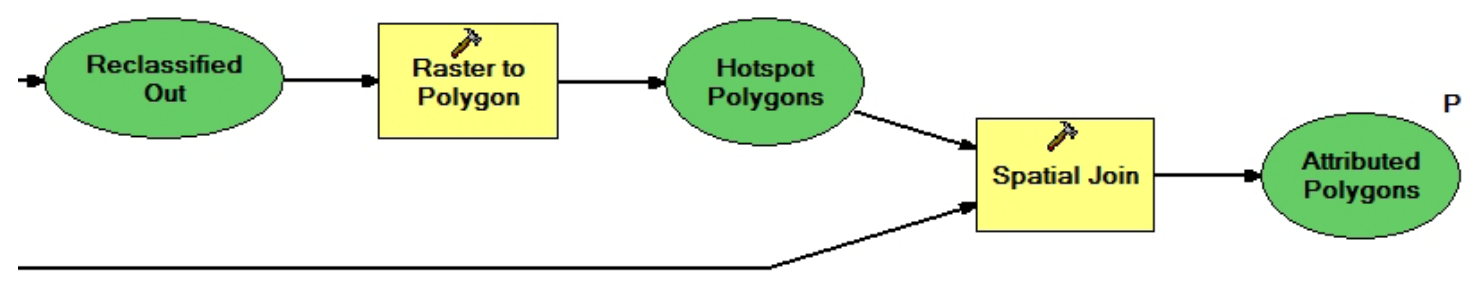

Figure 5.7 Adding Collision Attribute Data Step in the Model

\subsection{Hotspot Exploration Web Application}

After the hotspot detection model was implemented the web application was built to provide a user friendly interface for exploring the hotspot data and its attributes. The web application was coded in Flex using Adobe Flex Builder and implemented ESRI's ArcGIS API for Flex. The following sections discuss the data layers used in the web application, the tools created for the web application, and the output provided by the tools.

\subsubsection{Data Layers}

The first step in developing the web application was preparing the data for use by the application. This was accomplished by putting the data from the database and the results of the hotspot detection model into a map document. The map document was used to set up the display of the data and to set the symbology of the different feature classes. The hotspot polygons from the hotspot detection model and the traffic collision points were added to the map document first, but were set to not be visible. This was done so the web 
application could access the data but the data would not always be visible in the application. The hotspot polygon data was saved as graphic data in the application itself so the display and symbology could be managed in the web application. The traffic collision point data was queried by the tools in the application but wasn't made visible so as to reduce the cluttering of symbols in the application. The traffic signal and stop sign feature classes were then added to the map document. Both feature classes were given mimetic symbols to make them easily recognizable, since no legend was included in the web application. The display scale range was also limited for both of these feature classes so that they are only visible at certain scales. The traffic signal feature class was set to be visible at scales greater than 1:20,000 and the stop sign feature class was set to be visible at scales greater than 1:5,000. This reduced the cluttering of the symbols on the map since they are only visible when individual symbols can be distinguished. Once the map document was completed it was published for use by the web application. The data were published as a dynamic map service using ArcGIS Server, which allows the web application to display the data as they are symbolized in the map document and allows queries to be run on the data.

The web application also uses base maps provided by ESRI through ArcGIS Online. The free versions of the World Street Map and World Imagery base maps were used. A toolbar was created in the web application to provide the user a way to select between the two base maps using radio buttons, allowing only one base map to be displayed at a time.

\subsubsection{Application Startup}

A handful of tasks are completed when the application is first opened. First, the extent of the map is set to the extent of the City of Redlands. The map service is loaded into the map, as well as the two base map services and the World Street Map base map is selected as the visible base map. The hotspot polygons are also loaded into the map as graphics at startup, which requires a few different steps. First, a graphics layer is created to hold the hotspot polygons. A red simple fill symbol with an alpha value of 0.8 is applied to the graphics layer. The alpha value makes the polygons slightly transparent so that the base maps can be seen beneath them. Next, a query task is executed that runs a query which returns all of the hotspot polygons from the map service and puts them in the graphics layer. The hotspot polygons were put in a graphics layer instead of shown as part of the map service for several reasons. By using a graphics layer, the load placed on the server is reduced since the server is only queried once when the application is started. If the hotspot polygons were shown as a part of the map service then the server would be queried every time the map was updated from panning or zooming. Another reason the hotspot polygons were used as a graphics layer is that graphics layers can easily have different symbols applied to different graphics within a layer. This method was used to highlight a hotspot polygon when it is selected by the user.

\subsubsection{Hotspot Exploration Tools}

Eight different tools were created for the web application. Each of these tools looks at an attribute of the collisions that occurred within the selected hotspot and returns an output consisting of a table and a graph of the results. Each of the eight tools looks at a single attribute: month, time of day, severity, collision type, light condition, weather condition, 
number of people killed, and number of people injured. A toolbar was created to hold buttons for the tools. The buttons were programmed so that selecting a tool deselects all of the others, so that only one tool can be selected at a time. Each of the tools follows the same general functionality, outlined in Figure 5.8. A help button was also created for the application, which brings up a brief statement on the available functionality of the application.

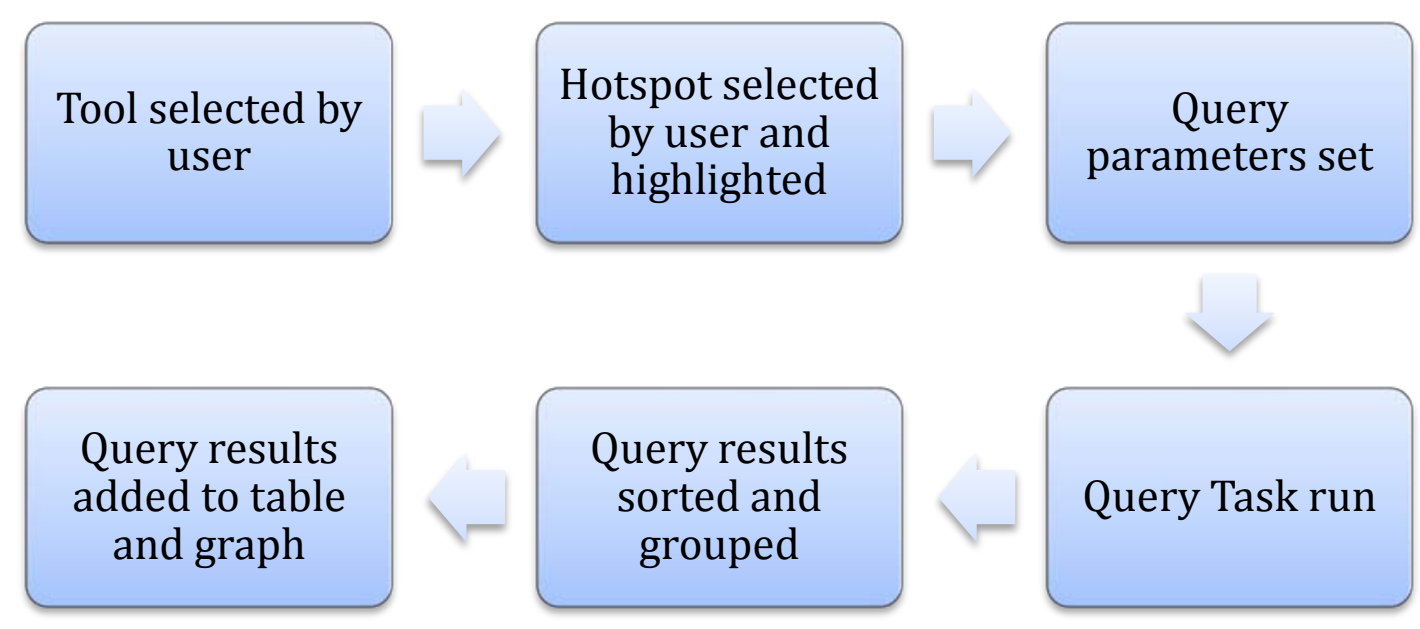

\section{Figure 5.8 Web Application Tool Procedures}

After the user has selected a tool they click on a single hotspot. The application then goes through each of the hotspots in the graphics layer to determine which one was selected. The selected hotspot is then highlighted by having its fill symbol changed from red to yellow, but the alpha value remains at 0.8 , as in the normal fill symbol. Next, the parameters for the query to be run by the tool are set. The spatial extent of the query is set to the extent of the selected hotspot polygon and the field to be returned is set to the field for the selected tool. The IncidentID field is also returned, since it is the default display field in the map service which is always returned by the query task. Once the parameters are set, the query task runs the query on the traffic collision layer in the map service and the results are returned as an array, which are then processed further so they can be put into a graphic output.

\subsubsection{Hotspot Exploration Tool Output}

The query results from each of the tools were put into both a table and a column chart, both of which are objects provided by Flex. The table and the column chart were put in a panel which is made visible after the query task is run. The header text for the panel was set to display the number of collisions that occurred in the hotspot selected by the user. The query results are returned in an array which allows them to be directly added to the table. The table has two columns: one for the IncidentID field and one for the field 
specified by the tool that the user selected. The column chart can only accept input as an array collection, which requires converting and grouping the data from the query results.

A function was created for each tool which takes the query results, groups the data by value, and populates the array collection that is the data source for the column chart with the grouped data. A single array collection was created and the functions change the values in the array collection based on the tool selected and the query results. The groupings used by each of the functions were determined first (Table 3). The month, severity, collision type, light condition, weather condition, number injured, and number killed fields each had a limited number of unique values, so they were grouped accordingly. The time of day was divided into three groups of eight hours each. The groups were spaced this way so one group would include collisions during the morning commute, the evening commute, and during the night. Once the groupings were determined, variables for each group were added to their functions. The functions then went through the query results and created counts for the number of collisions that fell into each group for the tool. The function then added the group names and their respective counts to the array collection. The titles for the $\mathrm{x}$ - and $\mathrm{y}$-axes were then added to the column chart to complete the function.

Table 3. Attribute Value Groupings for Tools

\begin{tabular}{|l|l|}
\hline Tool & Value Groups \\
\hline \hline Month & $\begin{array}{l}\text { January, February, March, April, May, June, July, } \\
\text { August, September, October, November, December }\end{array}$ \\
\hline Time of Day & 6:00-14:00, 14:00-22:00, 22:00-6:00 \\
\hline Severity & Property Damage, Injury, Fatality \\
\hline Collision Type & $\begin{array}{l}\text { Broadside, Head On, Hit Object, Other, } \\
\text { Overturned, Rear End, Side Swipe, Unknown, } \\
\text { Vehicle/Pedestrian }\end{array}$ \\
\hline Light Condition & $\begin{array}{l}\text { Daylight, Dark - Street Lights, Dark - No Street } \\
\text { Lights, Dark - Street Lights Not Functioning, } \\
\text { Dawn or Dusk }\end{array}$ \\
\hline Weather Condition & Clear, Cloudy, Fog, Raining, Windy, Other \\
\hline \# Killed & 0,1 \\
\hline \# Injured & $0,1,2,3,4,5$ or more \\
\hline
\end{tabular}




\subsection{Summary}

This chapter detailed the implementation of the hotspot detection model and the web application for examining the resulting hotspots. First, the creation of the model was discussed. The tools used by the model and the determination of the tool parameters were then considered. Next, the data used by the web application were examined. The programming methods implemented when the application starts were then detailed. Finally, the tools created for the application and their output were discussed. 


\section{Chapter 6 - Results and Analysis}

This chapter discusses the results of the hotspot detection model. The results of the model are compared against the collision point data and the attributes of the hotspot polygons are examined. The visual layout and the graphic output of the web application are also considered in this chapter. The implementation of the web application on the client's network is then detailed.

\subsection{Hotspot Detection Model Results}

The results of the hotspot detection model are shown in Figure 6.1. The model generated 59 different hotspot polygons which contain 863 of the 2,025 total traffic collision points. The hotspot polygons are dispersed throughout the city. The hotspots have a blocky appearance and many of them are rectangular, caused by the hotspots being converted to polygons from the kernel density raster. The raster grid cells that had values above the cutoff value determined in the reclassification step of the model were given a value of one. The Raster to Polygon tool converted all of the grid cells with a value of one into polygons. The tool combines any cells adjacent to another cell with the same value. This gives the polygons a blocky appearance since they are derived from groups of adjacent square grid cells.

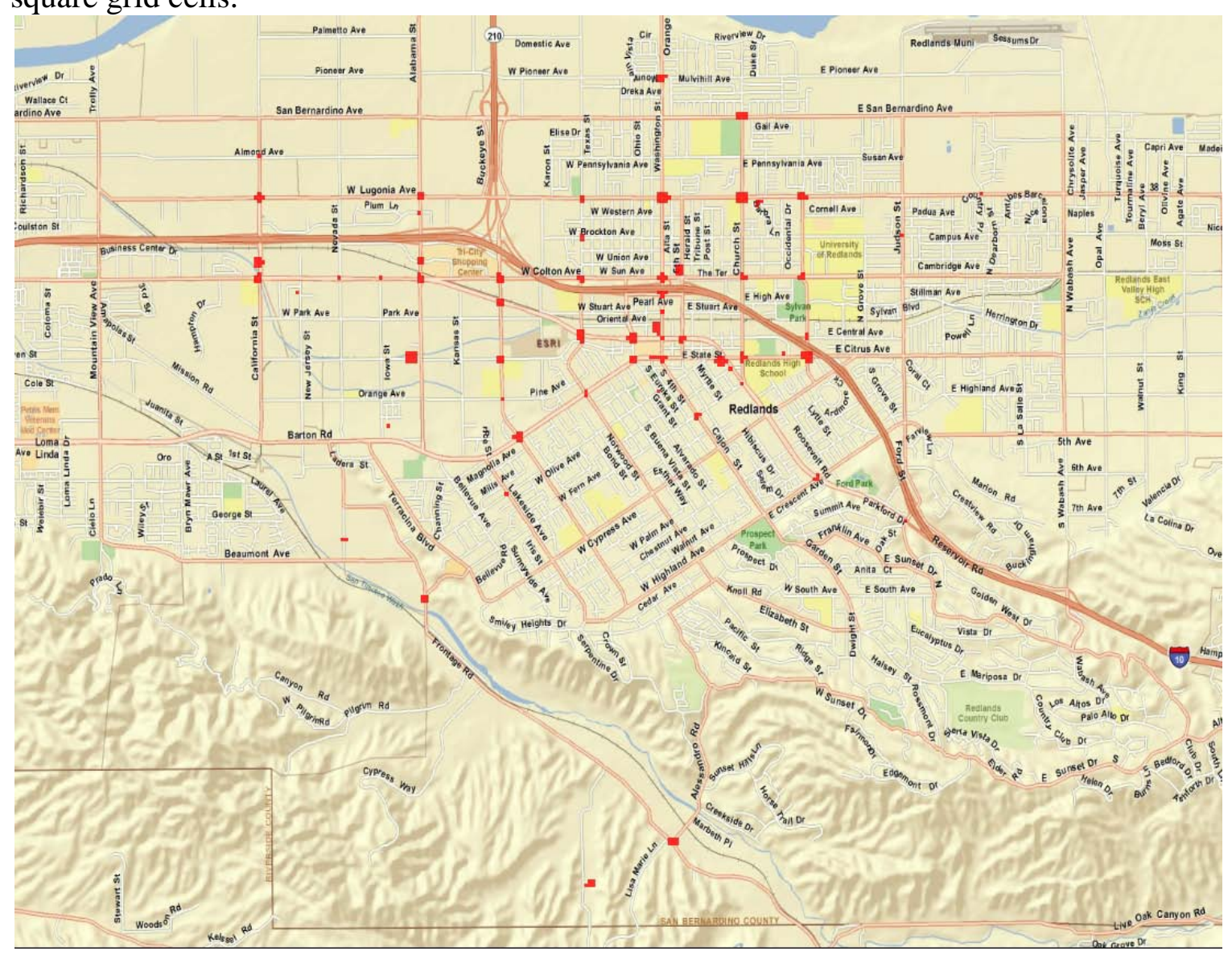

Figure 6.1 Hotspot Detection Model Results 
The location of the hotspot polygons were compared to the location of the collision points. All of the hotspot polygons have a large number of collision points at their center. The attribute table for the hotspot polygon feature class (Figure 6.2) shows that the hotspot polygons vary in the number of collision contained within them, with 7 being the lowest and 35 being the highest. This shows that the hotspot detection model did not create any hotspot polygons in areas with few or no traffic collision points. The attribute table in Figure 6.2 also shows that the area of a hotspot polygon generally increases as the number of collision that occurred in the polygon increases. This was expected because higher densities of traffic collision points will increase the density values calculated by the kernel for more of the grid cells surrounding the points. 


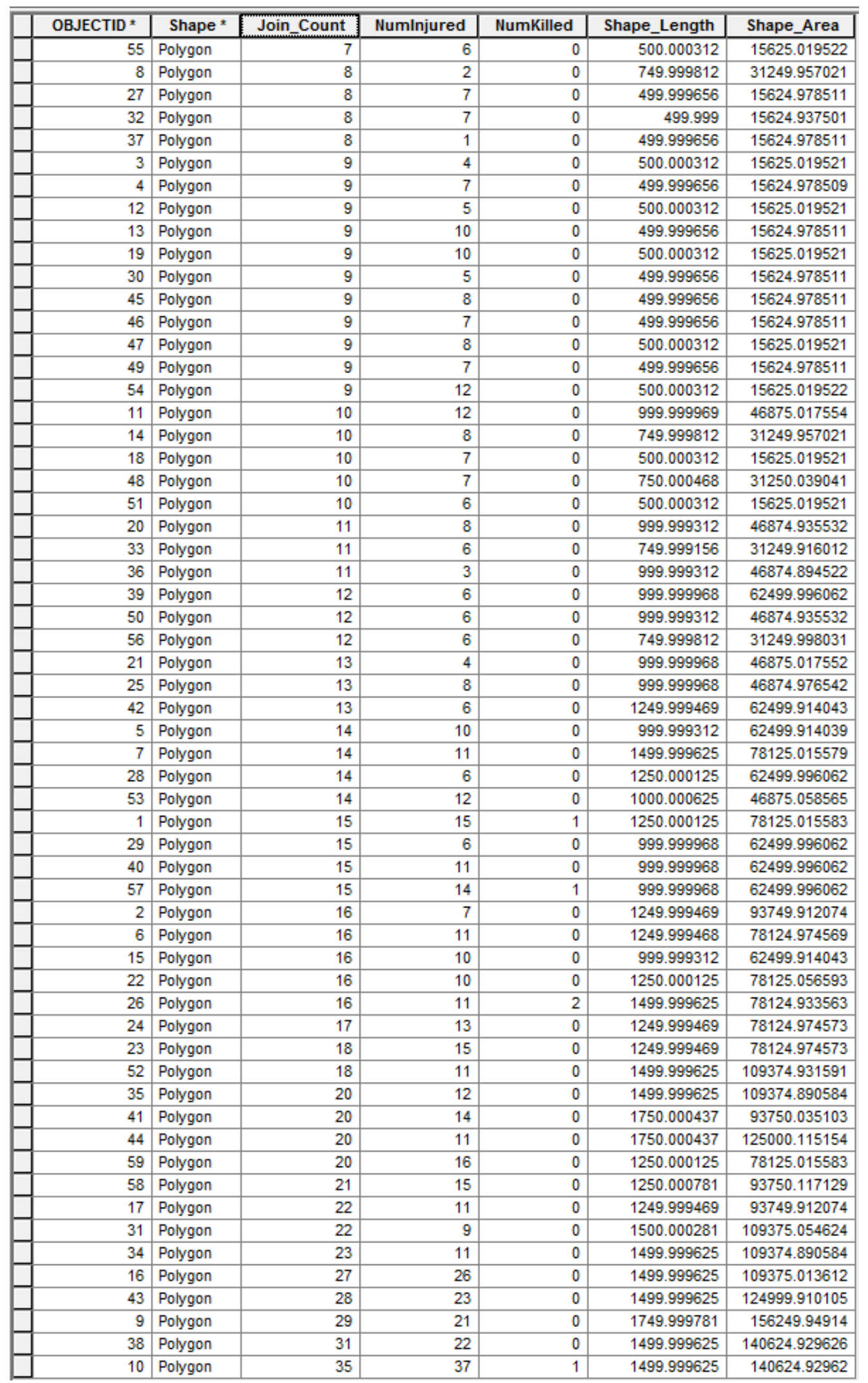

Figure 6.2 Hotspot Polygon Attribute Table 
One issue noted in the results was that four of the hotspot polygons did not cover any part of the road network. While many of the hotspot polygons include areas outside of the road network, they are still centered on the road network. These four hotspot polygons (Table 4) were examined further.

Table 4. Hotspot Polygons Not Covering Road Network

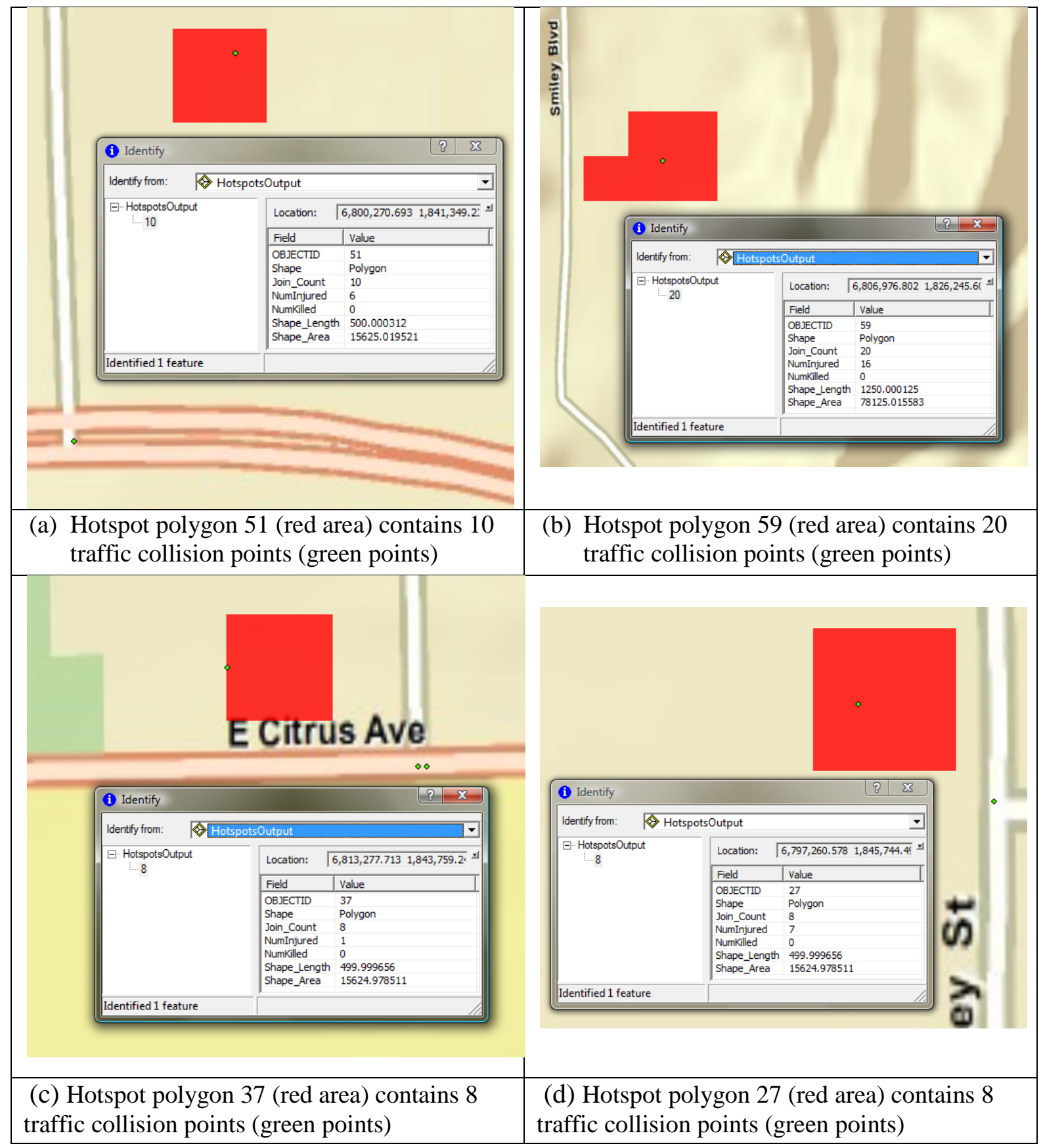


After examining the four hotspot polygons it was determined that the problem was not with the hotspot detection model but with the positional accuracy of the traffic collision points that were input to the model. The model accurately placed hotspots over areas with high densities of traffic collision points. The problem was that some of the traffic collision points were inaccurately located compared with the address listed in the attribute table. It was also noticed that the collision points with positional errors were all from the year 2007. After discussion with the client, it was found that the positional inaccuracies were caused by errors in the client's geocoding process. The client had switched to a different geocoding process in 2007 and the client believes that the points geocoded using the older process are the points with positional accuracy issues.

\subsection{Hotspot Exploration Web Application}

When the web application is first opened, the base map radio buttons, help button, and data tools are loaded. The two base maps from ArcGIS Online are also loaded and the Street Map base map is selected as the visible base map. Figure 6.3 shows the Street Map and Imagery base maps at the scale set when the application is first opened. The hotspot polygons are also queried and loaded into the graphics layer when the application is first opened. The graphics layer has a fill symbol applied to it which makes the hotspot polygons display in red with an alpha value of 0.8 , which makes them slightly transparent. This was done so that the roads could be seen beneath the hotspot polygons. 

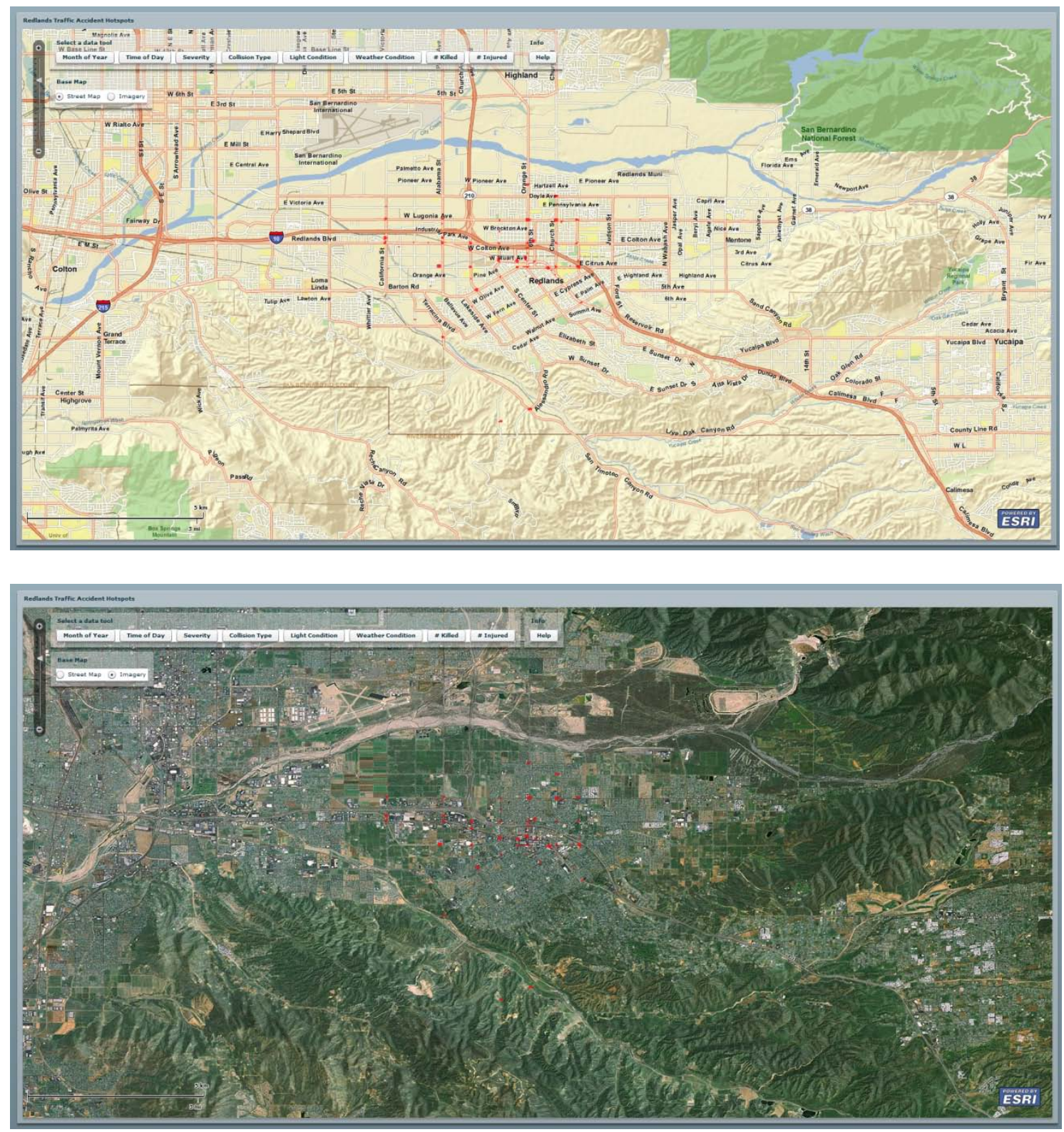

Figure 6.3 Web Application Base Maps

The traffic signals and stop signs are also displayed as part of the map service and have a limited display scale. The traffic signals were set to be at scales greater than 1:20,000 and the stop signs were set to be visible at scales greater than 1:5,000. These scales were chosen based on the scales that the tiled base maps display at. The traffic signals are visible at the largest three scales on the scale slider and the stop signs are only visible at the largest scale. This keeps the symbols from overlapping and obscuring each other or other map elements (Figure 6.4). 

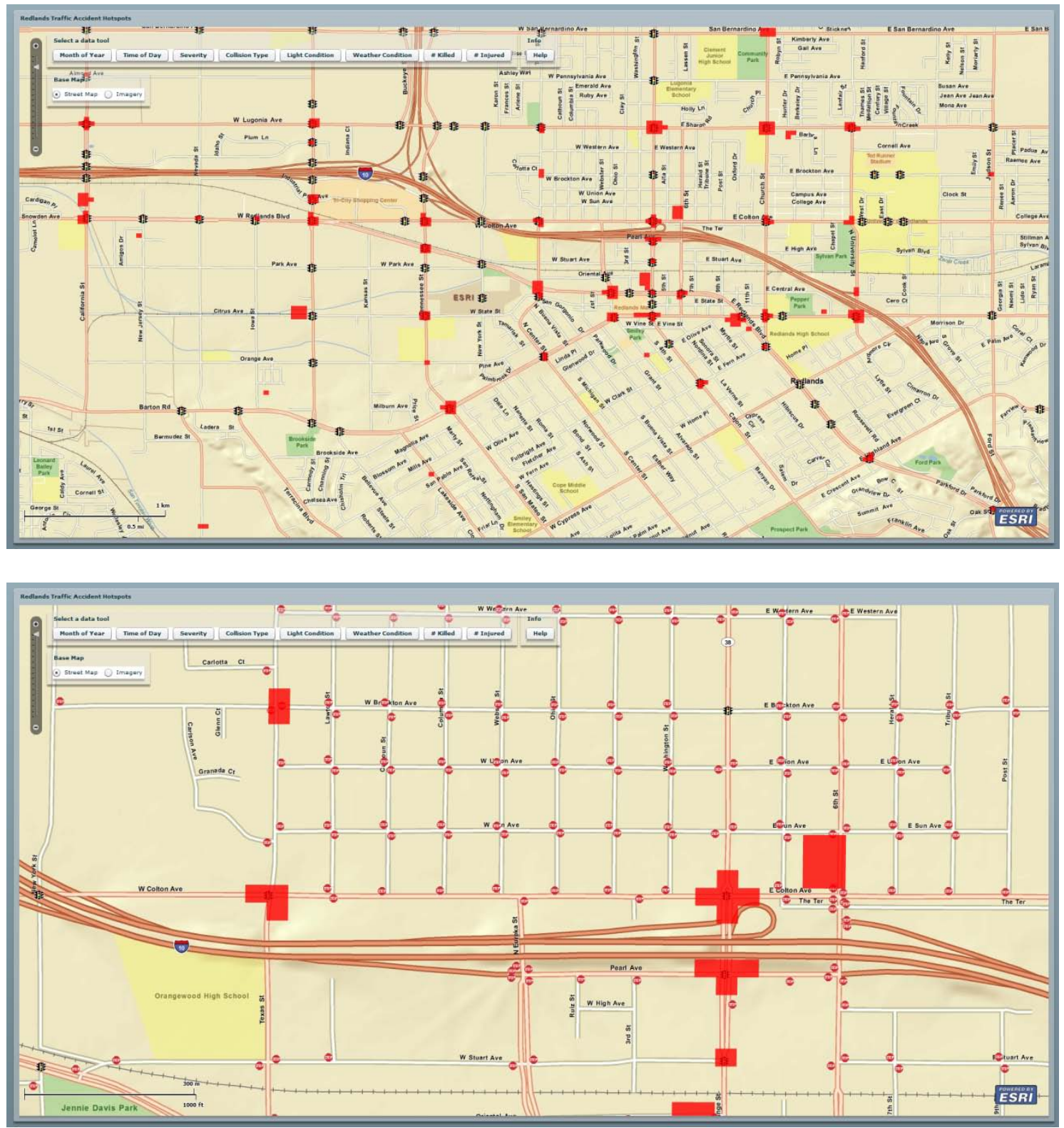

Figure 6.4 Traffic Signal and Stop Sign Display

The help button provided in the application brings up an alert box which gives a brief explanation of the functionality provided by the application (Figure 6.5). The zooming and panning capabilities described are the default ones provided by the ArcGIS API for Flex. 
Click on a tool then click on a red hotspot polygon

to display information about that hotspot. Use the

radio buttons to switch between base maps. Click

and drag to pan. Use the slider bar to zoom.

\section{OK}

\section{Figure 6.5 Help Dialog}

The tools provided each look at a single attribute of a selected hotspot polygon. First, the user selects one of the tools and then clicks on the hotspot polygon they want to examine. The selected hotspot polygon is then highlighted and the tool output is created. The graphic output of each tool is a table and a chart of the query results from the tool (Figure 6.6). The title of the panel containing the table and chart displays the number of collisions that occurred in the selected hotspot. The table contains the Incident ID of each collision that occurred in the selected hotspot, as well as the values for the attribute selected by the tool that was used. The results are grouped and sorted by attribute value and then added to the column chart. This illustrates the distribution of the attribute values of the collisions within the selected hotspot which can be useful in finding a common cause of collisions within a hotspot polygon.

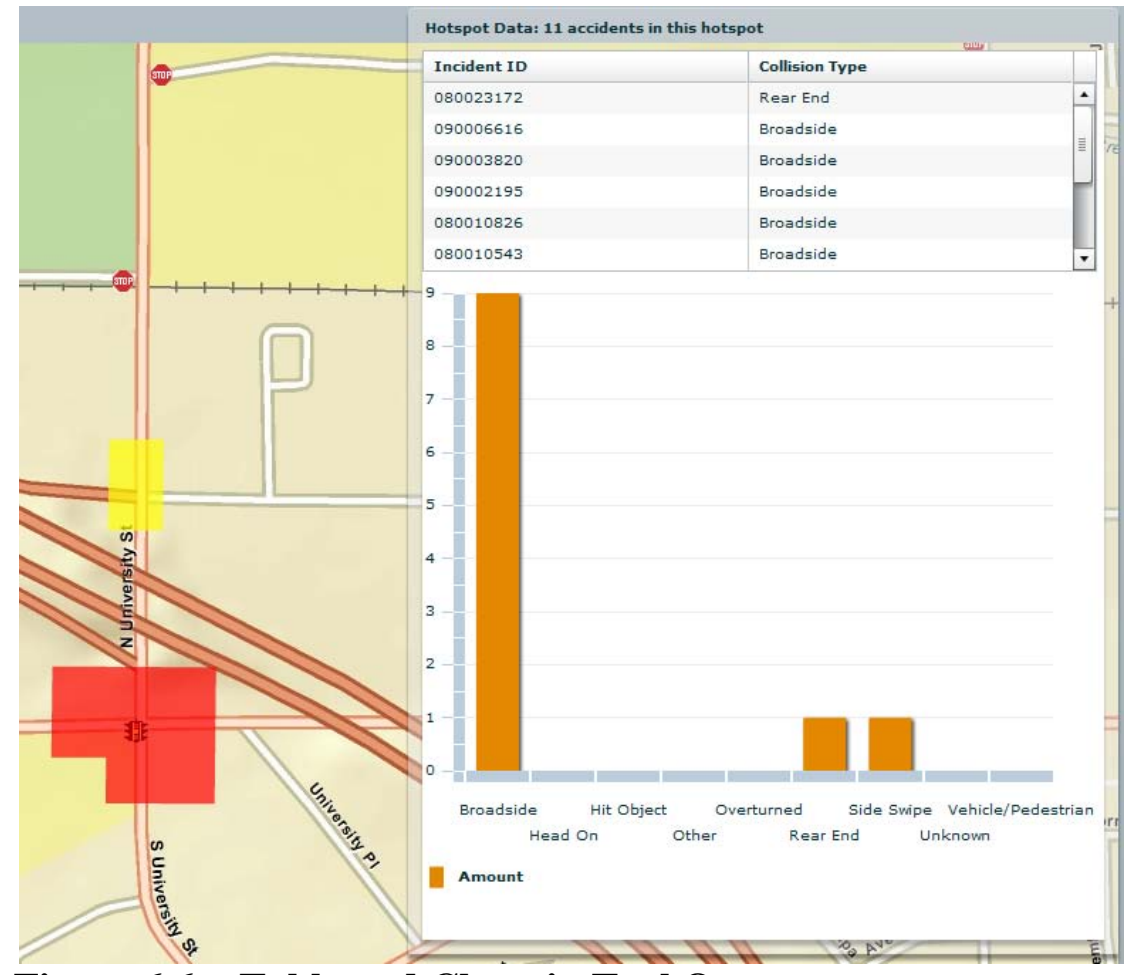

Figure 6.6 Table and Chart in Tool Output 


\subsection{Implementation on the Client's Network}

The final step in the project was implementing the web application on the client's network. This was a fairly straightforward process since the client already had the necessary software-ArcGIS Server-installed on its server. The file geodatabase and the map document were moved onto the server, links to the data in the map document were repaired, the map document was published as a map service on the client's server using ArcGIS Server, and the permissions were set for the published map service. With the map service published on the client's server, the links to the map service in the web application code were changed to point to the version of the client's server. The web application code was then compiled and moved to the web root directory on the server, making it available on the internet.

After the web application was implemented on the client's network it was tested to make sure no errors were introduced in the application during implementation. Each of the tools, the radio buttons for the base maps, and the help button was tested and no errors were found. A brief explanation of the functionality of the web application was given to Phil Mielke, the point of contact for the client. The final implemented version of the web application can be viewed at http://gis.cityofredlands.org/HotSpots/CityofRedlandsTrafficAccidentHotspotMap.html.

\subsection{Summary}

This chapter looked at the results of the hotspot detection model. The distribution and attributes of the hotspot polygons output by the model were discussed and the locations of the hotspot polygons were compared to the location of the traffic collision point data. Issues in the location of some the hotspot polygons were examined and found to be caused by inaccurate locations in some of the traffic collision points. The graphic display and functionality of the web application were then outlined. Finally, the implementation of the project data and web application on the client's network were detailed. 



\section{Chapter 7 - Conclusion and Future Work}

The final chapter looks at how the project accomplished the goals and objectives laid out in Chapter Three and examines the appropriateness of the analysis performed. Possible future work for the project is also discussed.

\subsection{Conclusion}

The overall goal for this project was to help the City of Redlands determine where to focus its traffic collision reduction efforts and help determine what measures should be implemented at those locations. This goal was achieved by the completion of the following objectives:

- Designing a database for the traffic collision data

- Creating a model to determine hotspots of traffic accidents

- Assigning attributes to the hotspots

- Creating a web application to view the hotspots

- Providing tools in the application for examining hotspot attribute data This project met the requirements determined in Chapter Three. The hotspot determination model creates hotspot polygons with collision data added to them using traffic collision point data as the only data input. The web application provides a simple and effective interface for viewing the hotspot polygons created by the model. The web application also includes tools for examining the attributes of the collisions that occurred within individual hotspot polygons. The web application was successfully implemented on the client's network and is available to the city staff and the public. These aspects meet the project goal of helping the City of Redlands determine where to focus its traffic collision reduction efforts and helping determine what measures should be implemented at those locations.

While the hotspot detection model created by this project met the goals of the project, other more appropriate methods for collision hotspot determination are available. Kernel density estimation (KDE) was used based on the client's request and information found in the literature review. KDE is useful for examining general point patterns, but can be problematic when used on collision points. KDE examines points in a 2dimensional space, while traffic collisions are limited to a road network. This means that $\mathrm{KDE}$ examines the density of collisions at many locations where no collisions are possible because the locations are not on the road network. This problem was increased in this project because KDE creates a rectangular grid and with the irregular shape of the City of Redlands, the number of grid cells outside of the road network was greatly increased. Another possible issue when using KDE with collision point data is that if the collision points at an intersection are dispersed around the intersection, they may fall into multiple grid cells and the density values calculated for those grid cells might not be high enough to consider the intersection a hotspot even though it has a high number of collisions. This was not an issue in this project since the collision points at each intersection were all coincident. This was caused by the collision points being geocoded to the intersections. Other methods for determining traffic collision hotspots are detailed in the following section. 


\subsection{Future Work}

\subsubsection{Accurate Traffic Collision Point Data}

The results of this project could be improved with the use of more accurate traffic collision point data. It was noted in Chapter Six of this report that some of the traffic collision points were inaccurately located when compared to the address attribute for the point. Going through each of the 2,025 traffic collision points to determine which ones were inaccurately located and moving them was beyond the scope of this project, so it was not done. If the traffic collision points were corrected, then the results of the hotspot detection model would be improved.

\subsubsection{Traffic Volume Data}

The inclusion of traffic volume data in the model would also greatly increase the relevance of the results. The number of traffic collisions on a road segment or at an intersection is related to the number of vehicles that travel through the road segment or intersection. Traffic collision hotspots are generally thought of as areas with a greater than average number of collisions. Traffic volume data could be used to determine what would be considered an average number of collisions for individual intersections and road segments. A major arterial road with ten collisions might not be a hotspot while a small residential road with ten collisions would definitely be a hotspot.

\subsubsection{Network Kernel Density Estimation}

Using kernel density estimation on a network would also greatly increase the accuracy of the results. Xie \& Yan (2008) present a method they call network kernel density estimation. They state that the kernel density estimation method implemented in ArcGIS is a planar method. Planar kernel density estimation views space as a 2-dimensional homogenous Euclidean space. They state that the problem with this method, when applied to traffic collisions, is that traffic collisions occur on roadways that are a part of a network and the assumption of a homogenous Euclidean space does not hold. Xie \& Yan suggest a kernel density estimation method that uses road segments in a network instead of grid cells. Their method also uses distance along the network between points instead of Euclidean distance. While this method is not currently available in ArcGIS, it could be programmed and implemented instead of the planar kernel density estimation used in the model in this project.

\subsubsection{Simulation to Determine Statistical Significance}

The analysis completed by the model in this project is exploratory in nature. It would be possible to determine the statistical significance of the hotspot detection model results through the use of simulation. Sabel et al. (2005) discuss using simulation to determine the statistical significance of kernel density estimation results. First, they used traffic volume data to determine the collision risk level of the road segments. They then used Monte Carlo simulation to determine statistical significance. Monte Carlo simulation creates a large number of randomly generated point patterns. The randomly generated 
patterns are limited to the road network and placement is weighted based on the collision risk level previously determined. The observed point pattern is then compared to the random patterns to determine if the observed pattern significantly differs from random. While this method is not currently available in ArcGIS, it could be programmatically created in the software. Applying this method to the project data would make the results much more robust.

\subsubsection{Additions to the Web Application}

The web application developed for this project provided tool that examine individual hotspots. It would also be useful to examine multiple hotspots at the same time. This could be accomplished by allowing the user to select more than one hotspot at a time and charting the values of the selected hotspots next to each other for comparison. Another method would be to provide a query tool that would select the hotspots to examine based on a user defined query. 



\section{Works Cited}

Adobe. (2009). Adobe - Flex 3: Features. Retrieved November 9, 2009, from http://www.adobe.com/products/flex/features/flex3/

Anderson, T. K. (2006). Comparison of spatial methods for measuring road accident 'hotspots': A case study of London. Journal of Maps, 55-63.

Anderson, T. K. (2009). Kernel density estimation and k-means clustering to profile road accident hotspots. Accident Analysis and Prevention, 41 (3), 359-364.

Brimicombe, A. J. (August 2005). Cluster detection in point event data having tendency toward spatially repetitive events. Proceedings of 8th International Conference on GeoComputation. Michigan.

Chainey, S., Tompson, L., \& Uhlig, S. (2008). The utility of hotspot mapping for predicting spatial patterns of crime. Security Journal, 21, 4-28.

City of Redlands Redevelopment Agency. (n.d.). Retrieved October 28, 2009, from City Demographics: http://www.redlandsrda.org/asp/site/about/citydemo/index.asp

Downs, J. A., \& Horner, M. W. (2007). Characterising linear point patterns. Proceedings of the GIScience Research UK Conference (GISRUK). Maynooth, Ireland.

Eck, J. E., Chainey, S. P., Cameron, J. G., Leitner, M., \& Wilson, R. E. (2005). Mapping crime: Understanding hot spots. USA: National Institute of Justice.

Grubesic, T. H., \& Murray, A. T. (December 2001). Detecting hot spots using cluster analysis and GIS. National Institute of Justice's Fifth Annual International Crime Mapping Research Conference. Dallas.

Lang, L. (1999). Transportation GIS. Redlands: Environmental Systems Research Institute, Inc.

McCullagh, M. J. (November 2006). Detecting hotspots in time and space. The 23rd meeting of the ISO/TC211. Riyadh.

Sabel, C., Kingham, S., Nicholson, A., \& Bartie, P. (2005). Road traffic accident simulation modelling - A kernel estimation approach. The 17th Annual Colloquium of the Spatial Information Research Centre University of Otago, (pp. 67-75). Dunedin, New Zealand.

Steenberghen, T., Dufays, T., Thomas, I., \& Flahaut, B. (2004). Intra-urban location and clustering of road accidents using GIS: A Belgian example. International Journal of Geographical Information Science, 18 (2), 169-181.

Williamson, D., McLafferty, S., McGuire, P., Mollenkopf, J., \& Goldsmith, V. (1999, January-March). A better method to smooth crime incident data. ArcUser Magazine, pp. 1-5.

Wolff, M., \& Asche, H. (April 2009). Exploring crime hotspots: Geospatial analysis and 3d mapping. Proceedings REAL CORP 2009, (pp. 147-156). Tagungsband.

Xie, Z., \& Yan, J. (2008). Kernel density estimation of traffic accidents in a network space. Computers, Environment and Urban Systems, 32 (5), 396-406. 



\section{Appendix A. Web Application Code}

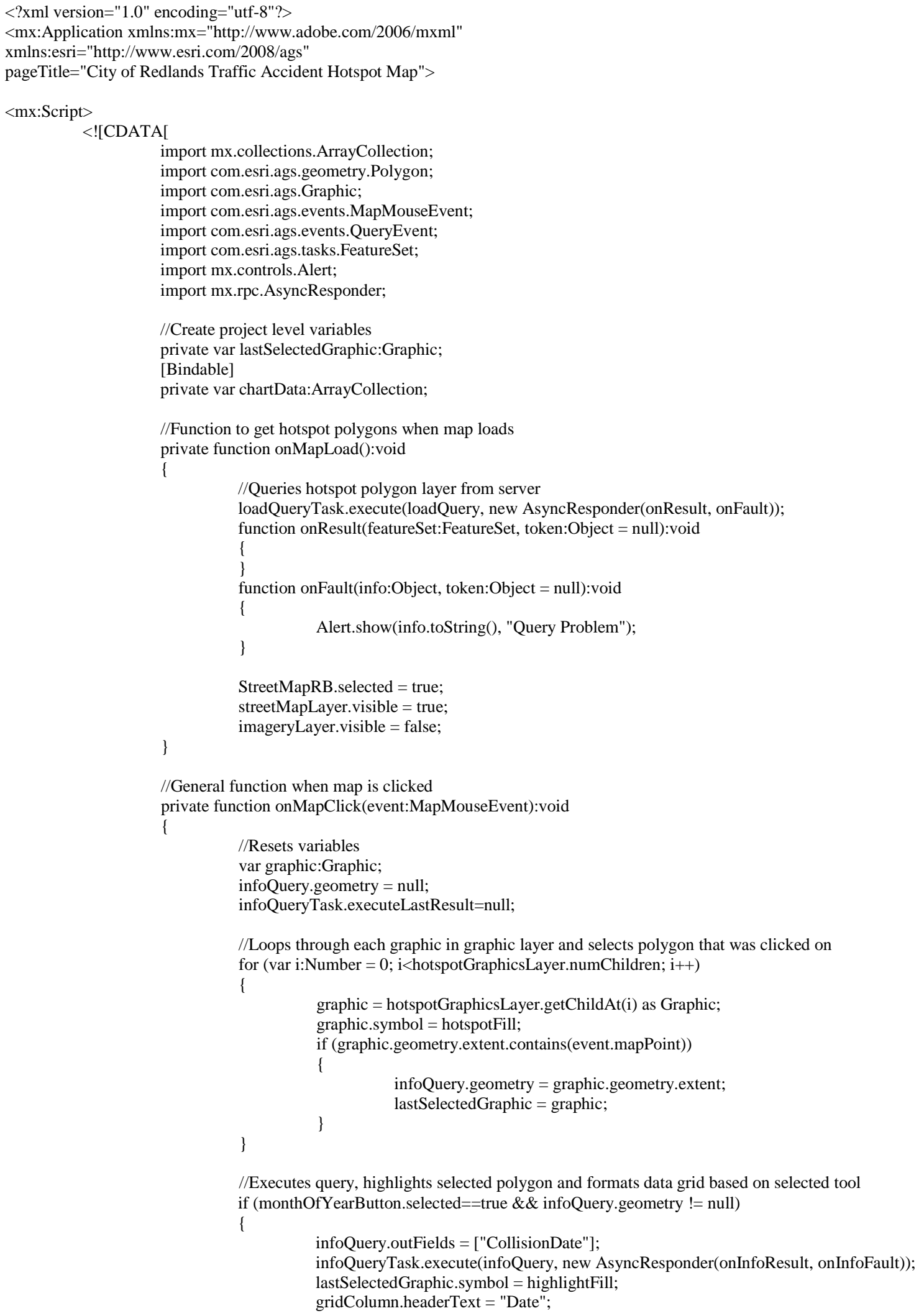




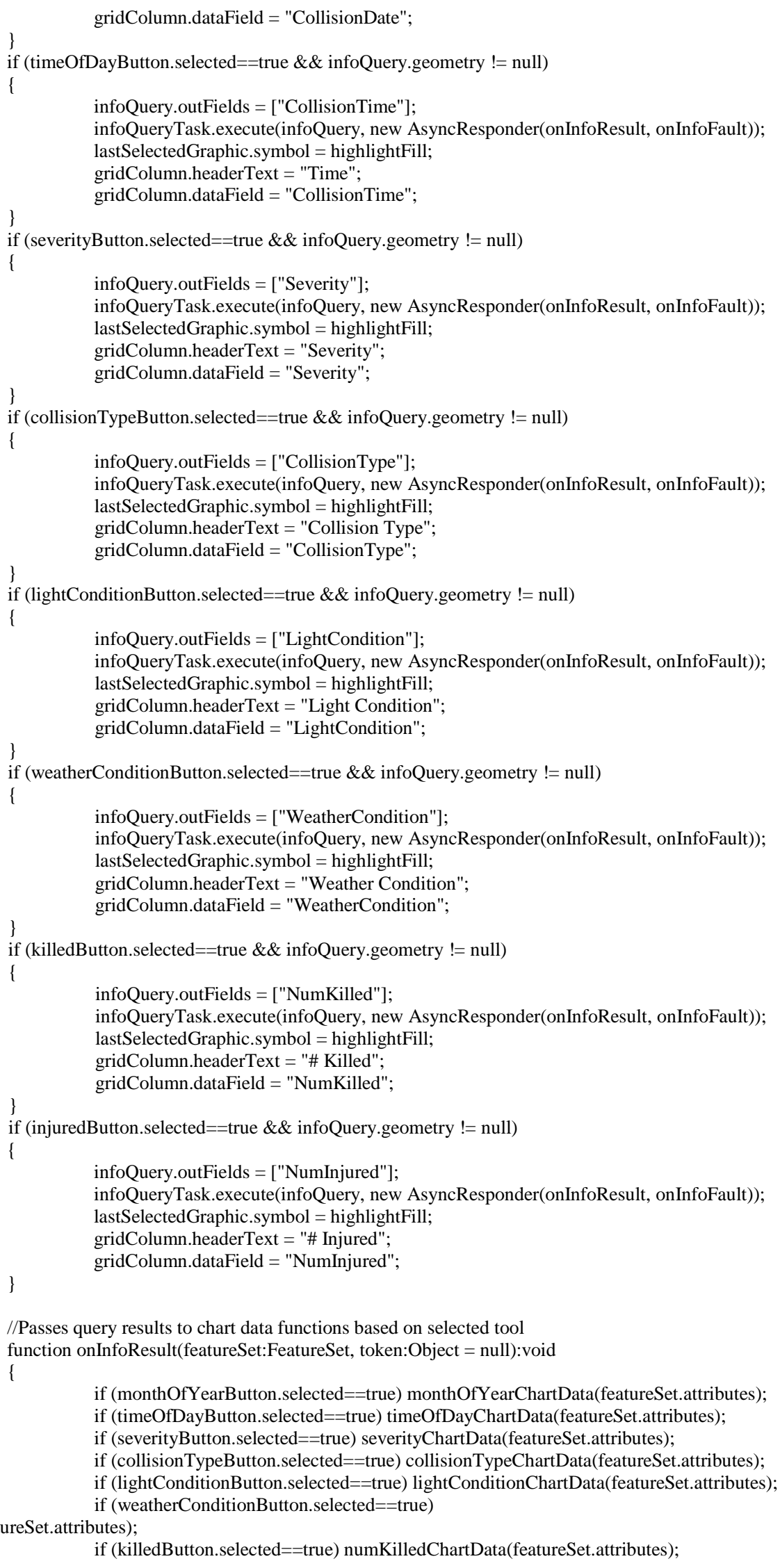




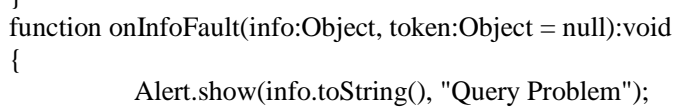

//Changes which base map is used based on radio button selected private function onRadioButtonClick(buttonName:String):void \{

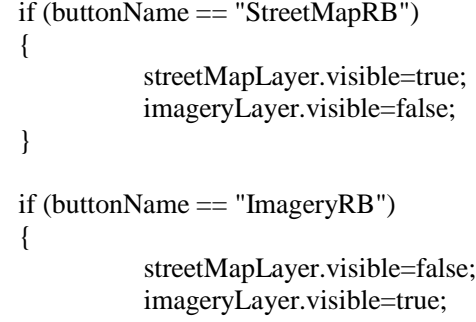

//Makes buttons act like radio buttons so only one tool can be selected at a time private function onButtonClick(buttonName:String):void

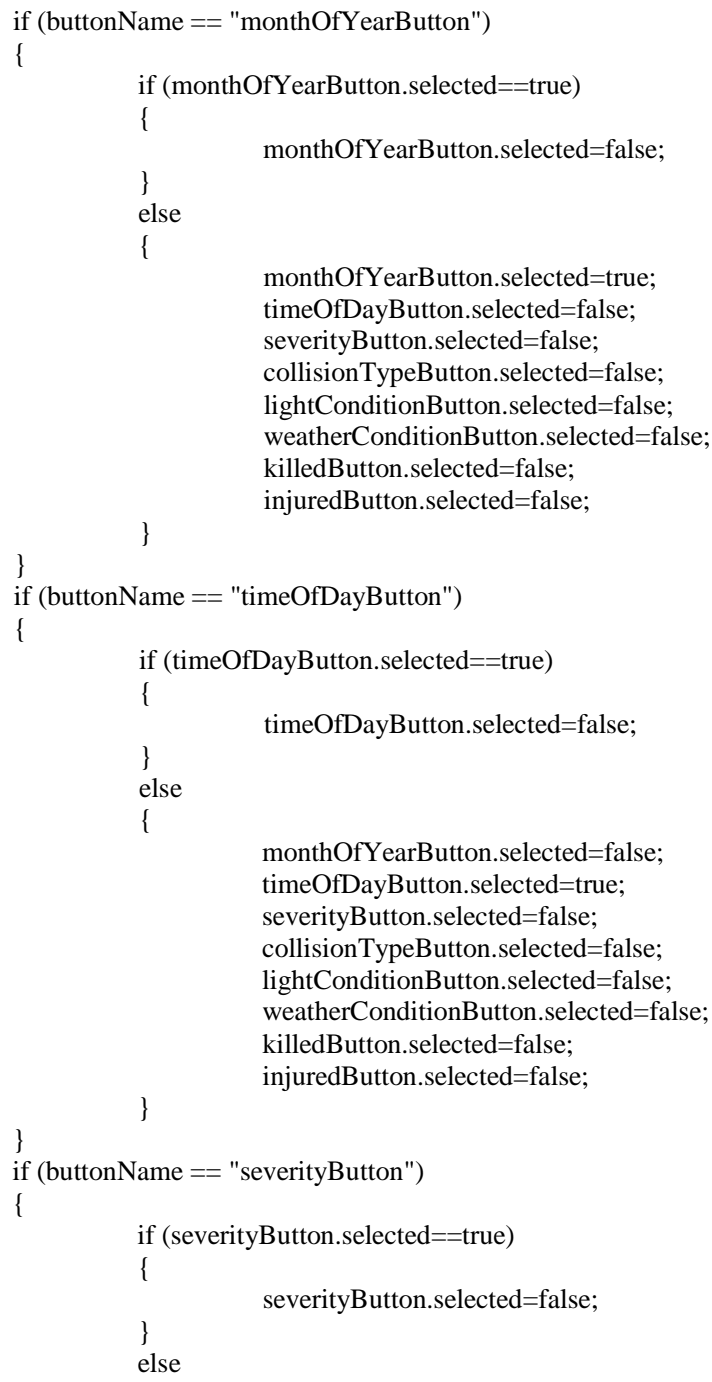


monthOfYearButton.selected=false;

timeOfDayButton.selected=false;

severityButton.selected=true;

collisionTypeButton.selected=false;

lightConditionButton.selected=false

weatherConditionButton.selected=false;

killedButton.selected=false;

injuredButton.selected=false;

\}

if (buttonName == "collisionTypeButton")

\{

f (collisionTypeButton.selected==true)

collisionTypeButton.selected=false;

else

\{

monthOfYearButton.selected=false; timeOfDayButton.selected=false; severityButton.selected=false; collisionTypeButton.selected=true; lightConditionButton.selected=false; weatherConditionButton.selected=false; killedButton.selected=false; injuredButton.selected=false;

if (buttonName == "lightConditionButton")

\{

if (lightConditionButton.selected==true)

\{

\}

lightConditionButton.selected=false;

else

\{

monthOfYearButton.selected=false; timeOfDayButton.selected=false; severityButton.selected=false; collisionTypeButton.selected=false; lightConditionButton.selected=true; weatherConditionButton.selected=false; killedButton.selected=false; injuredButton.selected=false;

\}

if (buttonName == "weatherConditionButton")

\{

if (weatherConditionButton.selected==true)

\{

\}

weatherConditionButton.selected=false;

else

\{

monthOfYearButton.selected=false; timeOfDayButton.selected=false; severityButton.selected=false; collisionTypeButton. selected=false; lightConditionButton.selected=false; weatherConditionButton.selected=true; killedButton.selected=false;

injuredButton.selected=false;

\}

if (buttonName == "killedButton")

\{

if (killedButton.selected==true)

\{

killedButton.selected=false; 
else

\{

monthOfYearButton.selected=false;

timeOfDayButton.selected=false;

severityButton.selected=false;

collisionTypeButton.selected=false;

lightConditionButton.selected=false;

weatherConditionButton.selected=false;

killedButton.selected=true;

\}

injuredButton.selected=false;

\}

if (buttonName == "injuredButton")

\{

if (injuredButton.selected==true)

\{

\}

injuredButton.selected=false;

else

\{

monthOfYearButton.selected=false;

timeOfDayButton.selected=false;

severityButton.selected=false;

collisionTypeButton.selected=false;

lightConditionButton.selected=false;

weatherConditionButton.selected=false;

killedButton.selected=false;

\}

injuredButton.selected=true;

\}

\}

//Chart data functions put query results into an array collection to be used by the column chart private function monthOfYearChartData(input:Array):void

\{

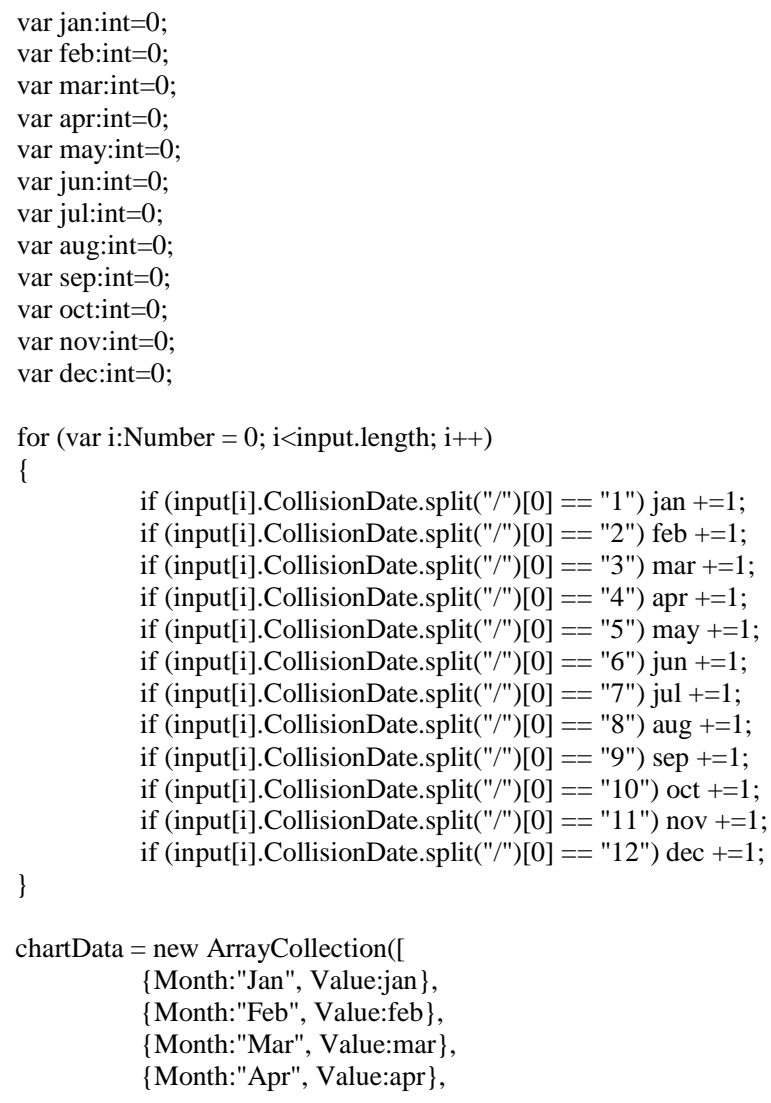




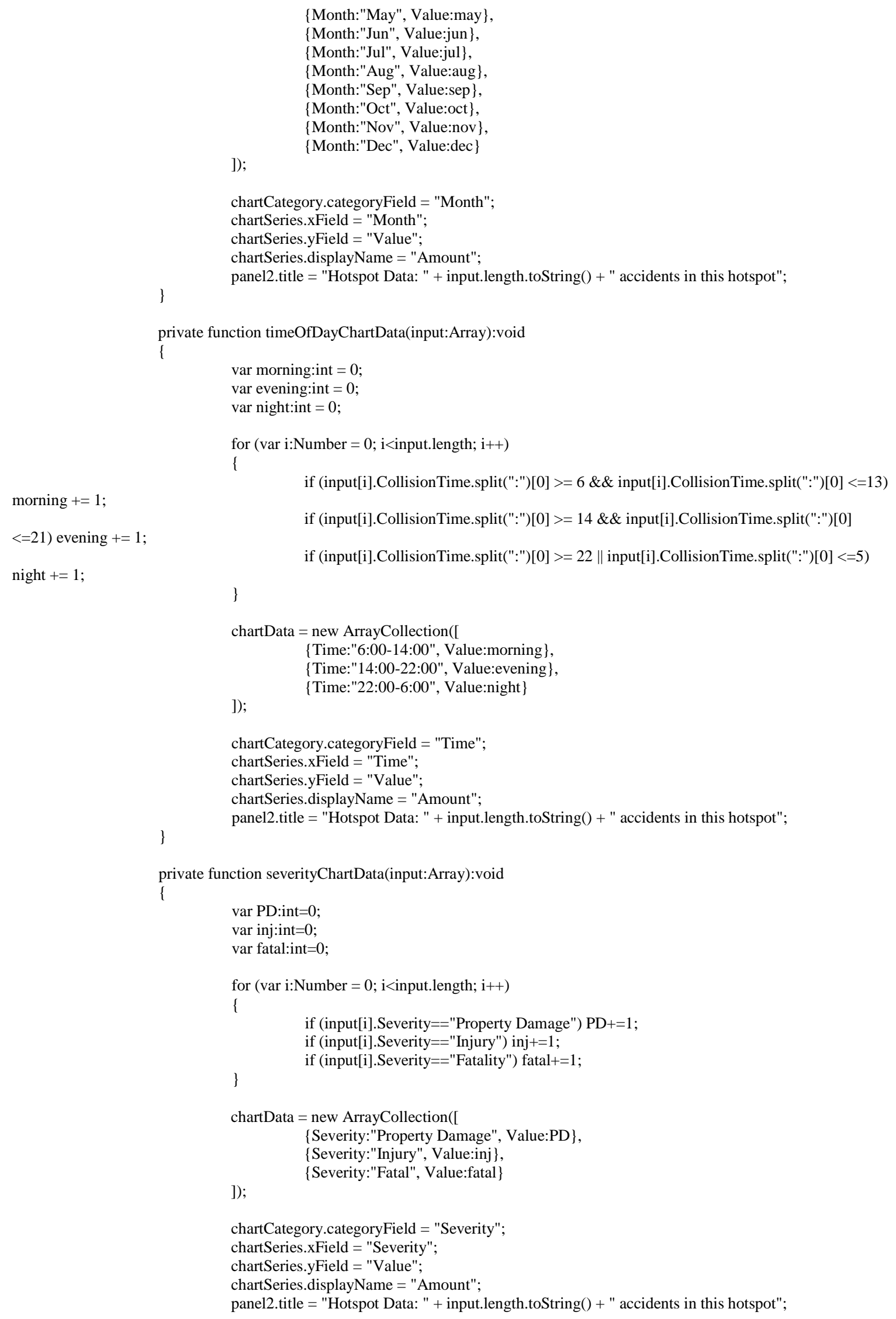


private function collisionTypeChartData(input:Array):void

\{

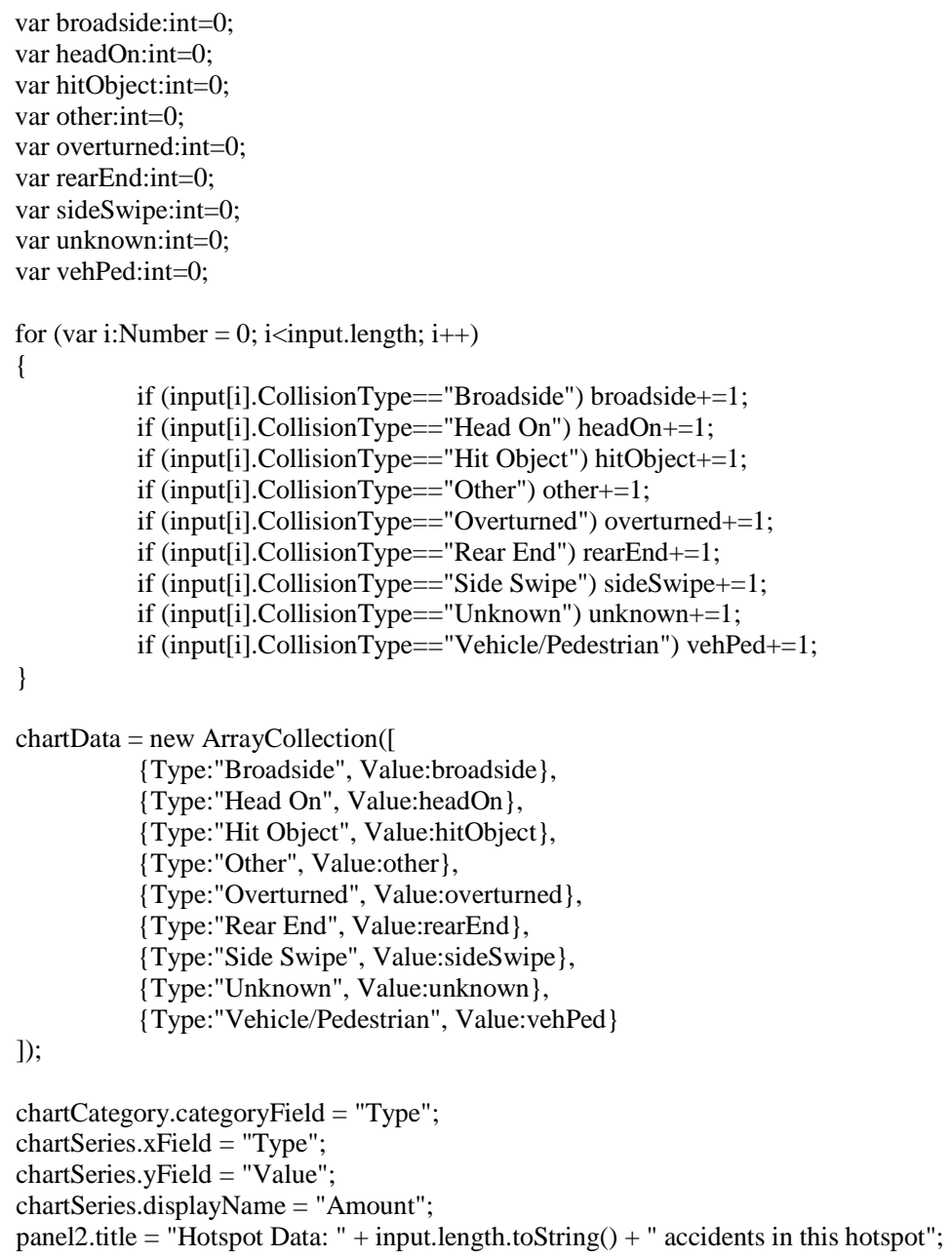


chartCategory.categoryField = "Condition";

chartSeries.xField = "Condition";

chartSeries.yField = "Value";

chartSeries.displayName = "Amount";

\}

panel2.title = "Hotspot Data: " + input.length.toString() + " accidents in this hotspot";

private function weatherConditionChartData(input:Array) :void

\{

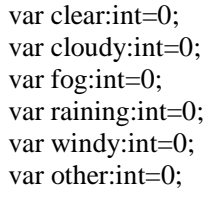

private function numInjuredChartData(input:Array):void

\{

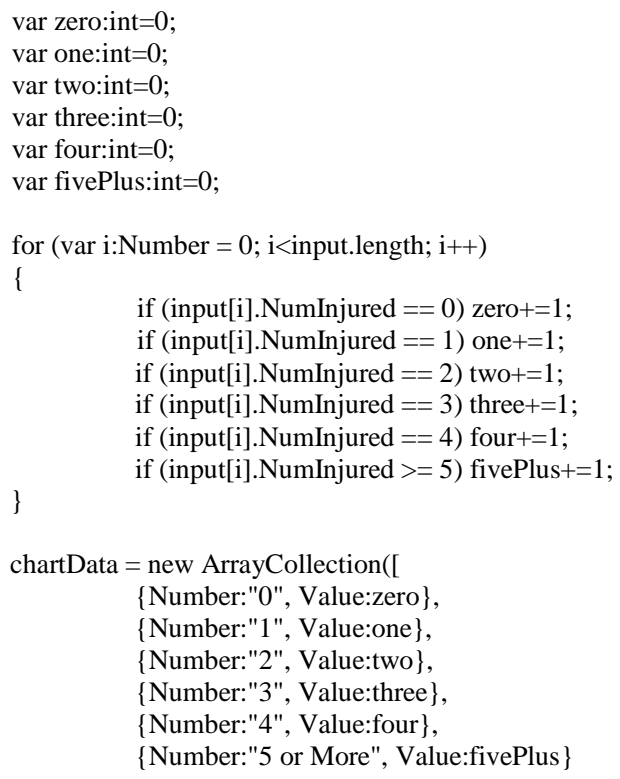


chartCategory.categoryField = "Number"; chartSeries.xField = "Number";

chartSeries.yField = "Value";

chartSeries.displayName = "Amount";

\}

panel2.title = "Hotspot Data: " + input.length.toString() + " accidents in this hotspot";

private function numKilledChartData(input:Array):void

\{

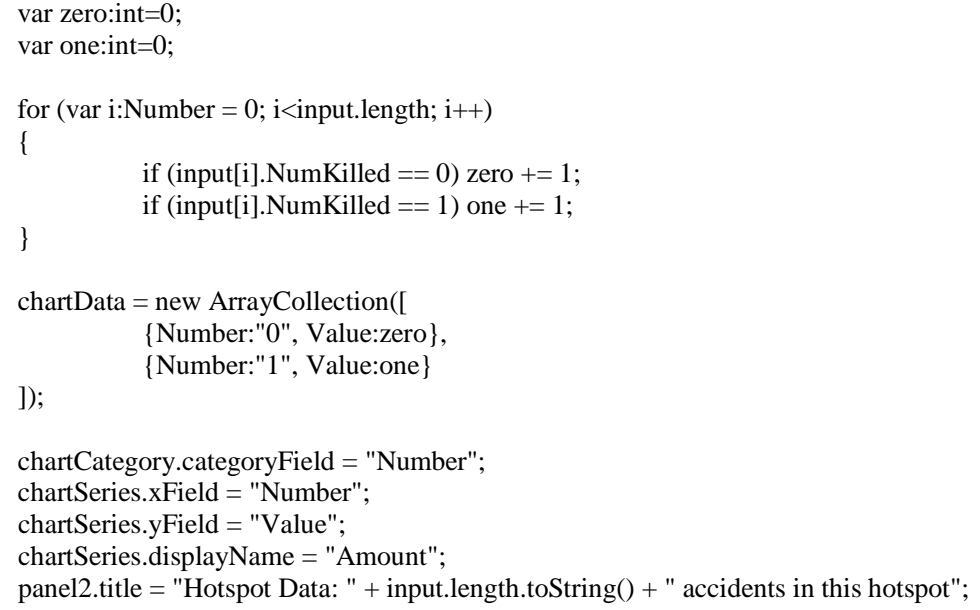

//Displays help instructions when help button is clicked private function onHelpButtonClick():void

\{

var info:String;

info = "Click on a tool then click on a red hotspot polygon to display information about that hotspot. Use the radio buttons to switch between base maps. Click and drag to pan. Use the slider bar to zoom.";

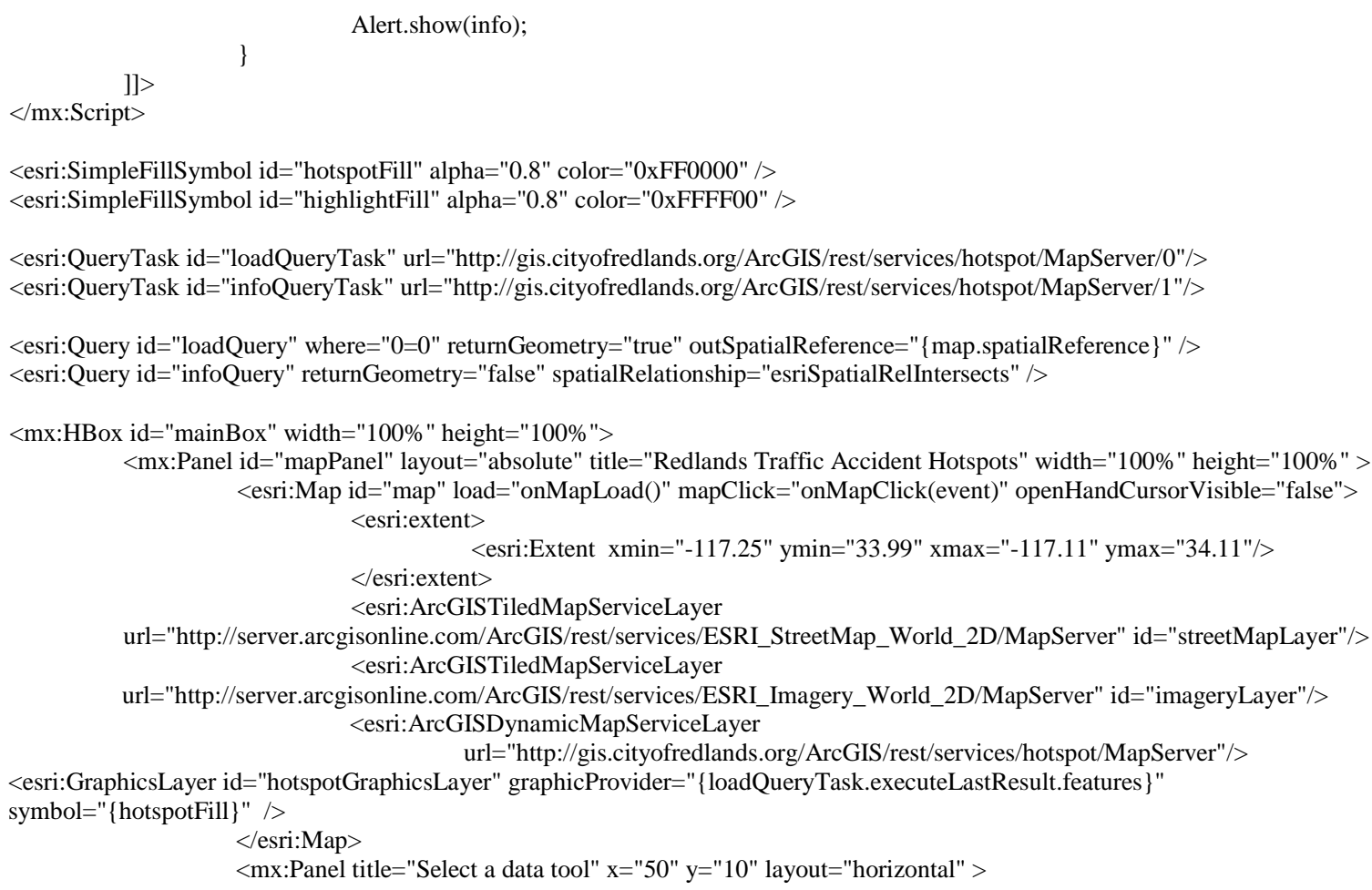


$<$ mx:Button id="monthOfYearButton" label="Month of Year"

click="onButtonClick('monthOfYearButton')"/>

$<$ mx:Button id="timeOfDayButton" label="Time of Day"

click="onButtonClick('timeOfDayButton')"/>

$<$ mx:Button id="severityButton" label="Severity" click="onButtonClick('severityButton')"/> $<$ mx:Button id="collisionTypeButton" label="Collision Type"

click="onButtonClick('collisionTypeButton')"/>

$<$ mx:Button id="lightConditionButton" label="Light Condition"

click="onButtonClick('lightConditionButton')" />

$<$ mx:Button id="weatherConditionButton" label="Weather Condition"

click="onButtonClick('weatherConditionButton')" / >

<mx:Button id="killedButton" label="\# Killed" click="onButtonClick('killedButton')"/>

$</$ mx:Panel $>$

$<\mathrm{mx}$ :Button id="injuredButton" label="\# Injured" click="onButtonClick('injuredButton')"/>

click="onRadioButtonClick('StreetMapRB')"/>

$<$ mx:Panel title="Base Map" layout="horizontal" x="50" y="80" >

click="onRadioButtonClick('ImageryRB')" />

$<$ mx:RadioButton id="StreetMapRB" label="Street Map"

$<$ mx:RadioButton id="ImageryRB" label="Imagery"

$</$ mx:Panel $>$

$<$ mx:Panel x="900" y="10" title="Info">

$</ \mathrm{mx}$ :Panel $>$

<mx:Button id="helpButton" label="Help" click="\{onHelpButtonClick()\}"/>

$<$ mx:Panel visible=" \{infoQueryTask.executeLastResult != null $\}$ " id="panel2" height="666" width="500" includeInLayout="false" $\mathrm{x}=$ " $\{$ map.width-500 $\}$ " $\mathrm{y}=$ "-25">

width="99\%">

<mx:DataGrid id="dataGrid" dataProvider="\{infoQueryTask.executeLastResult.attributes $\}$ "

$<\mathrm{mx}$ :columns $>$

<mx:DataGridColumn id="idColumn" headerText="Incident ID"

dataField="IncidentID"/>

categoryField="Number"/> $</ \mathrm{mx}$ :columns $>$

$<$ mx:DataGridColumn id="gridColumn"/>

$</$ mx:DataGrid $>$

$<$ mx:ColumnChart id="dataChart" width="99\%" dataProvider=" $\{$ chartData $\}$ " > $<$ mx:horizontalAxis >

$<\mathrm{mx}$ :CategoryAxis id="chartCategory" dataProvider=" $\{$ chartData $\} "$

$</$ mx:horizontalAxis $>$

$<$ mx:series $>$

$<$ mx:ColumnSeries id="chartSeries" xField="Number" yField="Value"

displayName="Amount"/>

$</$ mx:series $>$

$</ \mathrm{mx}$ :ColumnChart $>$

$<\mathrm{mx}$ :Legend dataProvider=" $\{$ dataChart $\} " />$

$$
</ \text { mx:Panel }>\text { Panel }>
$$

$</$ mx:HBox $>$

$</ \mathrm{mx}$ :Application $>$ 